\title{
A Mini-fluid Challenge of 200 mL Predicts fluid Responsiveness Using Pulmonary Artery Catheter in Septic Shock Patients an Observational Study
}

\author{
Ting Yang \\ Peking Union Medical College Hospital \\ Li Weng \\ Peking Union Medical College Hospital \\ Wei Jiang \\ Peking Union Medical College Hospital \\ Chunyao Wang \\ Peking Union Medical College Hospital \\ Jingmin Peng \\ Peking Union Medical College Hospital \\ Xiaoyun Hu \\ Peking Union Medical College Hospital \\ Shan Li \\ Peking Union Medical College Hospital \\ Bin Du ( $\square$ dubin98@gmail.com) \\ Peking Union Medical College Hospital
}

\section{Research}

Keywords: cardiac output (CO), mini-fluid challenge, fluid responsiveness (FR), septic shock

Posted Date: August 18th, 2020

DOI: https://doi.org/10.21203/rs.3.rs-56714/v1

License: (9) (1) This work is licensed under a Creative Commons Attribution 4.0 International License. Read Full License 


\section{Abstract}

Background: Mini-fluid challenge may predict fluid responsiveness and limit fluid overload. This study was designed to explore the minimal infusion volume in effectively predicting fluid responsiveness in septic shock patients.

Methods: ICU septic shock patients with indwelling pulmonary artery catheter received five sequential intravenous boluses of $100 \mathrm{~mL} 4 \%$ gelatin. Cardiac output was measured with thermodilution before fluid challenge (baseline) and three minutes after each bolus. Fluid responsiveness (FR) was defined as an increase in CO greater than $10 \%$ after $500 \mathrm{~mL}$ fluid infusion. The smallest volume which can perform an effective fluid challenge was analyzed.

Results: Forty-seven patients were included in this prospective study. After $500 \mathrm{~mL}$ volume expansion, thirty-six patients presented with FR $(77 \%)$ and 11 patients were fluid nonresponders. A mini fluid of $100 \mathrm{~mL}$ colloid had a poor predictive value (AUC $=0.67, p>0.05)$. The minimal volume required to predict FR in a mini fluid challenge was $200 \mathrm{~mL}$. An increase in CO greater than $5.2 \%$ after $200 \mathrm{~mL}$ colloid infusion was able to predict FR with a sensitivity of $83.3 \%$ and specificity of $90.9 \%$. The AUC under the ROC curve was $0.93(95 \% \mathrm{Cl}: 0.84-1$, $p<0.05)$.

Conclusion: In septic shock patients, a minimal volume of $200 \mathrm{~mL} 4 \%$ gelatin could reliably detect fluid responders and nonresponders.

Trial registration: ClinicalTrial.gov (NCT01941472). Registered on 13 September 2013.

\section{Background}

Fluid therapy is the cornerstone of septic resuscitation. According to the Early Goal Directed Therapy (EGDT) of sepsis bundle, an aggressive fluid resuscitation has been widely applied and known to improve survival in sepsis[1-4]. However, several retrospective studies have shown that $67 \%$ of patients with septic shock developed fluid overload on the first day of admission, with an average positive fluid balance of 4.2 L[5-8]. In recent decade, a restrictive fluid strategy has been applied to shock resuscitation for fewer complications and shorter hospital stays compared with a liberal fluid strategy[9-11]. Therefore, it is a reasonable step to assess the patient's response to fluid infusion to avoid excessive fluid administration[12, 13].

The Surviving Sepsis Campaign recommended dynamic cardiac measurements to guide fluid resuscitation in 2016. Mini-fluid challenge, passive leg raising (PLR) test and end-expiratory occlusion (EEO) test, all of these dynamic approaches allow the selection of fluid responders by inducible changes of hemodynamic parameters after changing the preload gaining popularity $[14,15]$. Mini-fluid challenge and PLR test and EEO test share the same principle, which can prevent patient from unnecessary fluid. These tests require a precise and real-time hemodynamic assessment to be properly interpreted. However, mini-fluid challenge can be used when other tests are not available and are not limited by the patient's disease condition and position, such as intra-abdominal hypertension, amputation, and prone position during therapy. Thus, mini-fluid challenge has attracted more attention from both ICU physicians and anesthetists.

The mini fluid challenge was firstly described by Muller et al. in 2011. They found an infusion of $100 \mathrm{~mL}$ colloid over 1 minute and the assessment by cardiac output using velocity time integral (VTi) at the aortic outflow tract could predict fluid responsiveness (FR). Since then, a total of seven investigations have been published [16-21]. Although a mini-fluid challenge may help the decision-making process of fluid management, the investigation results differed from each other, especially in minimal volume and cut off value of hemodynamic parameters change. Wu et al.reported that a minimal volume of $50 \mathrm{~mL}$ could predict FR using TTE to calculate VTi[19]. Muller et al. used $100 \mathrm{~mL}$ to predict FR also with VTi by TTE. Guinot.'s and Wang.'s work showed similar results with SV measured by thoracic impedance cardiography (ICG) and cardiac output (CO) measured by pulse contour (PiCCO). Meanwhile, Smorenberg's study showed that a better predictive minimal volume was $200 \mathrm{~mL}$ by applying pulse contour cardiac output measurement (PulseCO ${ }^{\mathrm{R}}$, COli) to calculate CO[16]. In addition to accessing the minimal volume by milliliter $(\mathrm{mL})$, Aya.et al[22] demonstrated that a dose of $4 \mathrm{~mL} / \mathrm{Kg}$ crystalloid could increase the transient stop-flow arm arterial-venous equilibrium pressure and detect FR. So here comes the question: what is the minimal infusion volume in effectively predicting fluid responsiveness. We noticed that when measuring $\mathrm{CO}$, the method of thermodilution by pulmonary artery catheter (PAC), which is the gold standard of $\mathrm{CO}$ measurement, was not used in these studies. Furthermore, the majority participants of the studies were perioperative patients in a stable hemodynamic state. Another question that needs to be answered is: whether mini-volume can perform a real change in septic shock patients. Therefore, it is necessary to explore the minimal volume in fluid challenge by PAC and test its reliability in detecting responders $(\mathrm{R})$ and nonresponders (NR).

In clinical practice, dynamic measurements are more recommended compared with static measurement. However, these measurements including $\mathrm{CO}$ monitor or echocardiograph require specific devices and skilled technicians. Therefore, a surrogate of $\mathrm{CO}$ which can be simply used and easily obtained to evaluate $\mathrm{FR}$ is needed. According to Fick equation, mixed venous oxygen saturation $\left(\mathrm{SvO}_{2}\right)$ is in the balance of 
cardiac index $(\mathrm{Cl})$, oxygen saturation and oxygen consumption[23]. We hypothesized that $\mathrm{SvO}_{2}$ can indirectly assess whether $\mathrm{CO}$ is sufficient enough to meet patient's enough. Based on the same hypothesis, the concept of finding parameters of oxygen metabolism to assess CO status seems to be reasonable and more convenient to apply to clinical settings. Central venous-to-arterial carbon dioxide partial pressure $\left(\mathrm{Pcv}-\mathrm{aCO}_{2}\right)$ and central venous oxygen saturation $\left(\mathrm{ScvO}_{2}\right)$ are two parameters expected to be effective in clinical practice. In this study, we attempt to observe changes in physiological parameters and evaluate their predictive abilities in mini fluid challenge[24-26].

\section{Methods \\ Study design}

This was a single-center, prospective observational pilot study. The clinical trial was registered on ClinicalTrial.gov (NCT01941472). The study protocol was approved by the Ethical Committee of Peking Union Medical College Hospital (ZS1085). Written informed consent was obtained from all patients or their legal representatives.

\section{Patients}

This study was conducted from July 2019 to July 2020. Patients over age of 18 years and under 80 years who have been diagnosed with septic shock and required fluid resuscitation were included. Septic shock in this study was defined in accordance with international criteria[3]. The exclusion criteria included other types of shock diagnosed, such as cardiogenic shock with evidence of acute coronary syndrome, chronic cardiac dysfunction, known allergy to colloid fluids, pregnancy, and simultaneous participation in another biomedical study. All patients received $\mathrm{CO}$ monitoring by thermodilution of PAC during their ICU stays, and the contraindications of PAC monitoring CO were excluded.

\section{Hemodynamic monitoring}

The arterial blood pressure was monitored from an arterial line (Becton Dickinson infusion therapy systems Linc., Utah, USA) placed in a radial artery or dorsalis pedis artery. PAC with 6 canals was placed in the internal jugular vein, and CO was calculated by the continuous thermodilution technique equipped with this PAC (Swan-Ganz CCOmbo CCO/SvO ${ }_{2}$, Vigilance II ${ }^{\text {TM }}$ monitor, Edwards Lifesciences, Irvine, CA, USA). All the mentioned above catheters connected to pressure transducers and the IntelliVue Patient Monitor MP70 (Philips Medical System, Boeblingen, Germany).

Mini-FC

Fluid challenge was performed in patients with hypotension (SBP $<90 \mathrm{mmHg}$ or MAP $<65 \mathrm{mmHg}$ ), or patients with evidences of tissue hypoperfusion (including but not limited to oliguria, skin mottling, altered mental status, cool peripheries, and hyperlactatemia, etc). This study consisted of six steps. The first set of measurements, including heart rate (HR), mean arterial pressure (MAP), pulmonary artery wedge pressure (PAWP), central venous pressure (CVP) and cardiac output (CO) as well as systemic vascular resistance (SVR) were recorded. CO values at each time-point were calculated from the average of three measured values. Baseline sets of blood gas were collected from arterial line (arterial blood gas), central venous in PAC canal (venous blood gas) and mixed venous canal in PAC canals (mixed venous blood gas) (S1). The first $100 \mathrm{ml}$ of $4 \%$ gelatin [Gelofusine; B. Braun Medical (Suzhou) Company Limited, Suzhou, China] was manually infused with a syringe of $60 \mathrm{~mL}$ ( $50 \mathrm{~mL}$ twice) within 2 minutes using. The second set of measurements and blood gas were then recorded immediately within 8 minutes after FC (including 3 minutes to calculate the $\mathrm{CO}$ and 5 minutes to obtain blood gas) (S2). Then, we repeated the colloid injection (100 $\mathrm{mL}$ within 2 minutes), hemodynamic data collection and blood gas sets collection (within 8minutes) as described above every 10 minutes for 4 times (S3-S6). All the patients received a total volume of $500 \mathrm{~mL}$ during 40 minutes, and 6 sets of data were analyzed after then. A brief study design is described in the Fig. 1. Patients were well sedated and there were no alterations in therapy during min-FC. The decision to optimize circulation was at the discretion of the physicians. A CO increase of more than $10 \%$ at the end of $500 \mathrm{~mL}$ volume expansion was defined as FR

\section{Statistical analysis}

Data were expressed as number, percentage, while continuous variables were expressed as the means \pm standard deviation (SD) or median with interquartile ranges (IQR) within 25-75\%, as appropriate. Before FC, patient characteristics between R and NR were compared using Student's T or Mann-Whitney U for continuous data and chi-square test for categorical data. A Student's paired t-test was used for withingroup comparisons (between S1 and S2, S3, S4, S5, S6). The difference between R and NR was compared by Student's t-test. The receiver operator characteristic (ROC) curves generated for $\Delta \mathrm{CO}_{100 \mathrm{~mL}}, \Delta \mathrm{CO}_{200 \mathrm{~mL}}, \Delta \mathrm{CO}_{300 \mathrm{~mL}}$ and $\Delta \mathrm{CO}_{400 \mathrm{~mL}}$, and the area under the ROC curve (AUROC) were calculated to assess predictive value. The Youden index was used to determine the optimal cutoff value of changes in cardiac output when the AUROC was greater than 0.5 . Spearman's correlation coefficient $r_{s}$ was used to quantify relations between continuous 
variables. Statistical analyses was performed with SPSS 25.0 software (SPSS, Inc, Chicago, IL), MedCalc (statistical software version 15.6 .1 for Windows) and GraphPad Prism 7. A value of $p<0.05$ was considered to be statistically significant.

\section{Results}

During the study period, 142 patients with acute circulatory failure were admitted from July 2019 to July 2020 during the study period. 95 patients who met the exclusion criteria were excluded, leaving 54 patients with septic shock from different sites of infection were eligible to the study. In addition, 7 patients had not been completed the study because of different reasons. Thus, 47 patients were finally included and analyzed. The detailed information of patient flow diagram was recorded in the flow chart (Fig. 2). Baseline demographic and clinical characteristics of participants between R and NR are shown in the Table 1. Baseline hemodynamics and blood oxygen metabolism indices were described and compared in Supplemental Table 2. Except for CO and DAP, there was not significant differences between R and NR at baseline.

Table 1

Baseline demographic and characteristics of participants

\begin{tabular}{|c|c|c|c|c|}
\hline Studied parameters & $\begin{array}{l}\text { Overall } \\
(n=47)\end{array}$ & $\begin{array}{l}R \\
(n=36)\end{array}$ & $\begin{array}{l}\text { NR } \\
(n=11)\end{array}$ & $P$ \\
\hline Age (years) & $58 \pm 15$ & $57 \pm 16$ & $59 \pm 9$ & 0.76 \\
\hline Weight (kg) & $64 \pm 12$ & $63 \pm 12$ & $69 \pm 13$ & 0.17 \\
\hline Height (cm) & $166 \pm 7$ & $166 \pm 7$ & $168 \pm 8$ & 0.24 \\
\hline BSA & $1.8 \pm 0.17$ & $1.78 \pm 0.17$ & $1.87 \pm 0.17$ & 0.13 \\
\hline Sex (male/female) & $27 / 20$ & $18 / 18$ & $9 / 2$ & 0.16 \\
\hline Temperature $\left({ }^{\circ} \mathrm{C}\right)$ & $37.9 \pm 0.8$ & $37.8 \pm 0.9$ & $38 \pm 0.8$ & 0.34 \\
\hline APACHE II & $24 \pm 8$ & $24 \pm 8$ & $23 \pm 8$ & 0.71 \\
\hline SOFA & $12 \pm 3$ & $13 \pm 3$ & $12 \pm 3$ & 0.6 \\
\hline Vasopressor, $\mathrm{n}$ & 47 & 36 & 11 & 0.2 \\
\hline Noradrenaline, $\mathrm{n}$ & 47 & 36 & 11 & 0.2 \\
\hline Dose, mcg/min & $36(18-70)$ & $36(19-60)$ & $70(18-96)$ & \\
\hline Adrenaline, $n$ & 1 & 1 & & \\
\hline Dose, $\mathrm{mcg} / \mathrm{min}$ & & 2 & & \\
\hline \multicolumn{5}{|l|}{ Infection sites } \\
\hline Thoracic & 23 & 16 & 6 & \\
\hline \multicolumn{5}{|l|}{ empyema } \\
\hline Abdominal & 15 & 4 & 2 & \\
\hline Cholangitis or cholecystitis Gastroenteritis & 4 & 5 & 3 & \\
\hline \multirow[t]{2}{*}{ Peritonitis } & 7 & 2 & & \\
\hline & 4 & & & \\
\hline Cavity Blood & 4 & 4 & 0 & \\
\hline Others & 5 & 4 & 1 & \\
\hline
\end{tabular}


Table 2

Baseline clinical characteristics comparison between responders and non-responders

\begin{tabular}{|c|c|c|c|c|}
\hline Studied parameters & $\begin{array}{l}\text { Overall } \\
(n=47)\end{array}$ & $\begin{array}{l}R \\
(n=36)\end{array}$ & $\begin{array}{l}\text { NR } \\
(n=11)\end{array}$ & $P$ \\
\hline HR (bpm) & $117 \pm 25$ & $118 \pm 26$ & $116 \pm 22$ & 0.97 \\
\hline $\mathrm{SAP}(\mathrm{mmHg})$ & $115 \pm 18$ & $116 \pm 19$ & $111 \pm 15$ & 0.34 \\
\hline $\mathrm{DAP}(\mathrm{mmHg})$ & $62 \pm 8$ & $63 \pm 8$ & $54 \pm 4$ & $<0.05$ \\
\hline $\mathrm{MAP}(\mathrm{mmHg})$ & $78 \pm 8$ & $79 \pm 8$ & $75 \pm 5$ & 0.06 \\
\hline CVP (mmHg) & $11 \pm 5$ & $10 \pm 3$ & $12 \pm 5$ & 0.29 \\
\hline $\mathrm{PAWP}(\mathrm{mmHg})$ & $14 \pm 4$ & $14 \pm 5$ & $14 \pm 4$ & 0.86 \\
\hline $\mathrm{CO}(\mathrm{L} / \mathrm{min})$ & $6.6 \pm 2.4$ & $6.2 \pm 2.4$ & $8.0 \pm 1.8$ & $<0.05$ \\
\hline $\mathrm{SV}(\mathrm{mL})$ & $58 \pm 23$ & $55 \pm 23$ & $70 \pm 17$ & 0.06 \\
\hline SVR (dynes $\cdot s \cdot \mathrm{cm}^{-5}$ ) & $976 \pm 515$ & $1051 \pm 522$ & $733 \pm 426$ & 0.2 \\
\hline $\mathrm{HBG}(\mathrm{g} / \mathrm{dL})$ & $9.4 \pm 2.5$ & $9.5 \pm 2.6$ & $9.5 \pm 2.6$ & 0.99 \\
\hline $\mathrm{ScvO}_{2}(\%)$ & $71.7 \pm 10.7$ & $71.2 \pm 10.7$ & $72.5 \pm 11.5$ & 0.74 \\
\hline $\mathrm{SvO}_{2}(\%)$ & $68.6 \pm 9.2$ & $68.2 \pm 9$ & $70.7 \pm 10$ & 0.45 \\
\hline $\mathrm{Lac}(\mathrm{mmol} / \mathrm{L})$ & $2.8(1.4-4.0)$ & $2.5(1.5-3.2)$ & $3(1.4-4.2)$ & 0.94 \\
\hline
\end{tabular}

\section{Hemodynamic parameters change with mini-fluid challenge}

Of the 47 patients, 36 (77\%) were FR to the FC. Hemodynamic variables with mini FC and their changes from the baseline are presented in the Table 3 and Table 4. The tendency of $\mathrm{CO}$ and $\Delta \mathrm{CO} \%$ after mini-fluid challenge in R and NR are shown in Fig. 3,4. After a volume expansion of $200 \mathrm{~mL}$ colloid, $\triangle \mathrm{CO} \%$ was significantly greater with $\mathrm{R}$ compared to $\mathrm{NR}(p<0.05)$. HR decreased after fluid infusion in both $\mathrm{R}$ and NR, whereas SAP, DAP, MAP, CVP and PAWP all showed an upward trend in both R and NR, except for baseline of DAP, there was no difference between the R and NR groups. 
Table 3

Hemodynamic variables before and after fluid challenge in R and NR.

\begin{tabular}{|c|c|c|c|c|c|c|c|c|c|c|}
\hline Group & $\begin{array}{l}\text { Infusion } \\
(\mathrm{mL})\end{array}$ & $\begin{array}{l}\mathrm{CO} \\
(\mathrm{L} / \mathrm{min})\end{array}$ & $\begin{array}{l}\text { SV } \\
(\mathrm{mL})\end{array}$ & $\begin{array}{l}\text { HR } \\
\text { (bpm) }\end{array}$ & $\begin{array}{l}\text { SAP } \\
(\mathrm{mmHg})\end{array}$ & $\begin{array}{l}\text { DAP } \\
(\mathrm{mmHg})\end{array}$ & $\begin{array}{l}\text { MAP } \\
(\mathrm{mmHg})\end{array}$ & $\begin{array}{l}\text { CVP } \\
(\mathrm{mmHg})\end{array}$ & $\begin{array}{l}\text { PAWP } \\
(\mathrm{mmHg})\end{array}$ & $\begin{array}{l}\text { SVR } \\
\left(\text { dynes } \cdot \mathrm{s} \cdot \mathrm{cm}^{-5}\right)\end{array}$ \\
\hline \multirow{6}{*}{$\begin{array}{l}\text { Responders } \\
(n=36)\end{array}$} & Baseline & $\begin{array}{l}6.2 \pm 2.4 \\
b\end{array}$ & $55 \pm 23$ & $118 \pm 26$ & $116 \pm 19$ & $63 \pm 8^{b}$ & $79 \pm 8^{b}$ & $10 \pm 3$ & $14 \pm 5$ & $1051 \pm 522^{b}$ \\
\hline & 100 & $\begin{array}{l}6.5 \pm 2.6 \\
a\end{array}$ & $\begin{array}{l}58 \pm 24 \\
a\end{array}$ & $116 \pm 26^{a}$ & $122 \pm 21^{a}$ & $64 \pm 9$ & $82 \pm 10^{a}$ & $11 \pm 3^{a}$ & $15 \pm 5^{a}$ & $1026 \pm 505^{b}$ \\
\hline & 200 & $\begin{array}{l}6.9 \pm 2.7 \\
a\end{array}$ & $\begin{array}{l}63 \pm 26 \\
a\end{array}$ & $\begin{array}{l}114 \pm 26 \\
a\end{array}$ & $122 \pm 28$ & $65 \pm 8^{a}$ & $84 \pm 9^{a}$ & $12 \pm 4^{a}$ & $16 \pm 5^{a}$ & $983 \pm 471^{b}$ \\
\hline & 300 & $\begin{array}{l}7.2 \pm 2.7 \\
a\end{array}$ & $\begin{array}{l}66 \pm 26 \\
a\end{array}$ & $\begin{array}{l}112 \pm 29 \\
a\end{array}$ & $\begin{array}{l}128 \pm 22 \\
a\end{array}$ & $67 \pm 10^{a}$ & $86 \pm 11^{a}$ & $13 \pm 4^{a}$ & $16 \pm 5^{a}$ & $954 \pm 454^{b}$ \\
\hline & 400 & $\begin{array}{l}7.4 \pm 2.8 \\
a\end{array}$ & $\begin{array}{l}67 \pm 26 \\
a\end{array}$ & $\begin{array}{l}113 \pm 26 \\
a\end{array}$ & $\begin{array}{l}130 \pm 23 \\
a\end{array}$ & $67 \pm 9^{a}$ & $87 \pm 11^{a}$ & $13 \pm 4^{a}$ & $17 \pm 5^{a}$ & $930 \pm 425^{b}$ \\
\hline & 500 & $\begin{array}{l}7.7 \pm 2.6 \\
a\end{array}$ & $\begin{array}{l}69 \pm 25 \\
a\end{array}$ & $\begin{array}{l}114 \pm 25 \\
a\end{array}$ & $\begin{array}{l}132 \pm 25 \\
a\end{array}$ & $68 \pm 10^{a}$ & $88 \pm 12^{a}$ & $14 \pm 4^{a}$ & $17 \pm 5^{a}$ & $889 \pm 389$ \\
\hline \multirow{6}{*}{$\begin{array}{l}\text { Nonresponders } \\
(n=11)\end{array}$} & Baseline & $\begin{array}{l}8.0 \pm 1.8 \\
\text { b }\end{array}$ & $70 \pm 17$ & $116 \pm 22$ & $111 \pm 15$ & $54 \pm 4^{b}$ & $75 \pm 5^{b}$ & $12 \pm 5$ & $14 \pm 4$ & $733 \pm 426^{b}$ \\
\hline & 100 & $8.1 \pm 2.0$ & $74 \pm 23$ & $114 \pm 22$ & $116 \pm 20$ & $61 \pm 6$ & $78 \pm 7^{a}$ & $13 \pm 5$ & $15 \pm 5$ & $666 \pm 188^{b}$ \\
\hline & 200 & $7.8 \pm 2.1$ & $72 \pm 21$ & $\begin{array}{l}114 \pm 21 \\
a\end{array}$ & $119 \pm 21$ & $61 \pm 6^{a}$ & $79 \pm 8^{a}$ & $13 \pm 5^{a}$ & $16 \pm 5^{a}$ & $703 \pm 212^{b}$ \\
\hline & 300 & $8.0 \pm 1.8$ & $73 \pm 20$ & $\begin{array}{l}114 \pm 22 \\
a\end{array}$ & $\begin{array}{l}122 \pm 22 \\
a\end{array}$ & $62 \pm 8^{a}$ & $80 \pm 9^{a}$ & $14 \pm 5^{a}$ & $17 \pm 4^{a}$ & $704 \pm 208^{b}$ \\
\hline & 400 & $8.0 \pm 2.0$ & $72 \pm 21$ & $\begin{array}{l}114 \pm 22 \\
a\end{array}$ & $121 \pm 25$ & $63 \pm 8^{a}$ & $82 \pm 10^{a}$ & $15 \pm 5^{a}$ & $18 \pm 4^{a}$ & $702 \pm 220^{b}$ \\
\hline & 500 & $7.9 \pm 1.9$ & $71 \pm 21$ & $\begin{array}{l}113 \pm 22 \\
a\end{array}$ & $\begin{array}{l}125 \pm 25 \\
a\end{array}$ & $63 \pm 8^{a}$ & $82 \pm 10^{a}$ & $15 \pm 5^{a}$ & $18 \pm 4^{a}$ & $720 \pm 223$ \\
\hline
\end{tabular}

Abbreviations: CO, cardiac output; SV, stroke volume; HR, heart rate; SAP, systolic arterial pressure; DAP, diastolic arterial pressure; MAP, mean arterial pressure; CVP, central venous pressure; PAWP, pulmonary artery wedge pressure; PAWP, pulmonary artery wedge pressure; SVR, systemic vascular resistance. ${ }^{a} P<0.05$ between baseline and volune expansion from $100 \mathrm{~mL}$ to $500 \mathrm{~mL}$ in subgroup; ${ }^{\text {b }} P<0.05$ between responders and nonresponders. 
Table 4

Changes in hemodynamics between $\mathrm{R}$ and NR

\begin{tabular}{|c|c|c|c|c|c|c|c|c|c|c|}
\hline Group & $\begin{array}{l}\text { Infusion } \\
(\mathrm{mL})\end{array}$ & $\begin{array}{l}\mathrm{CO} \\
(\mathrm{L} / \mathrm{min})\end{array}$ & $\begin{array}{l}\text { SV } \\
(\mathrm{mL})\end{array}$ & $\begin{array}{l}\text { HR } \\
\text { (bpm) }\end{array}$ & $\begin{array}{l}\text { SAP } \\
(\mathrm{mmHg})\end{array}$ & $\begin{array}{l}\text { DAP } \\
(\mathrm{mmHg})\end{array}$ & $\begin{array}{l}\text { MAP } \\
(\mathrm{mmHg})\end{array}$ & $\begin{array}{l}\text { CVP } \\
(\mathrm{mmHg})\end{array}$ & $\begin{array}{l}\text { PAWP } \\
(\mathrm{mmHg})\end{array}$ & $\begin{array}{l}\text { SVR } \\
\text { (dynes } \cdot \mathrm{s} \cdot \mathrm{cm}^{-} \\
\text {5) }\end{array}$ \\
\hline \multirow{6}{*}{$\begin{array}{l}\text { Responders } \\
(n=36)\end{array}$} & Baseline & $\begin{array}{l}6.2 \pm 2.4 \\
b\end{array}$ & $\begin{array}{l}55 \pm \\
23\end{array}$ & $\begin{array}{l}118 \pm \\
26\end{array}$ & $\begin{array}{l}116 \pm \\
19\end{array}$ & $63 \pm 8^{b}$ & $79 \pm 8^{b}$ & $10 \pm 3$ & $14 \pm 5$ & $1051 \pm 522^{b}$ \\
\hline & 100 & $\begin{array}{l}6.5 \pm 2.6 \\
a\end{array}$ & $\begin{array}{l}58 \pm \\
24^{a}\end{array}$ & $\begin{array}{l}116 \pm \\
26^{a}\end{array}$ & $\begin{array}{l}122 \pm \\
21^{a}\end{array}$ & $64 \pm 9$ & $\begin{array}{l}82 \pm 10 \\
a\end{array}$ & $11 \pm 3^{a}$ & $15 \pm 5^{a}$ & $1026 \pm 505^{b}$ \\
\hline & 200 & $\begin{array}{l}6.9 \pm 2.7 \\
a\end{array}$ & $\begin{array}{l}63 \pm \\
26^{a}\end{array}$ & $\begin{array}{l}114 \pm \\
26^{a}\end{array}$ & $\begin{array}{l}122 \pm \\
28\end{array}$ & $65 \pm 8^{a}$ & $84 \pm 9^{a}$ & $12 \pm 4^{a}$ & $16 \pm 5^{a}$ & $983 \pm 471^{b}$ \\
\hline & 300 & $\begin{array}{l}7.2 \pm 2.7 \\
a\end{array}$ & $\begin{array}{l}66_{ \pm}^{ \pm} \\
26^{a}\end{array}$ & $\begin{array}{l}112 \pm \\
29^{a}\end{array}$ & $\begin{array}{l}128 \pm \\
22^{a}\end{array}$ & $\begin{array}{l}67 \pm 10 \\
a\end{array}$ & $\begin{array}{l}86 \pm 11 \\
a\end{array}$ & $13 \pm 4^{a}$ & $16 \pm 5^{a}$ & $954 \pm 454^{b}$ \\
\hline & 400 & $\begin{array}{l}7.4 \pm 2.8 \\
a\end{array}$ & $\begin{array}{l}67 \pm \\
26^{a}\end{array}$ & $\begin{array}{l}113 \pm \\
26^{a}\end{array}$ & $\begin{array}{l}130 \pm \\
23^{a}\end{array}$ & $67 \pm 9^{a}$ & $\begin{array}{l}87 \pm 11 \\
a\end{array}$ & $13 \pm 4^{a}$ & $17 \pm 5^{a}$ & $930 \pm 425^{b}$ \\
\hline & 500 & $\begin{array}{l}7.7 \pm 2.6 \\
a\end{array}$ & $\begin{array}{l}69 \pm \\
25^{a}\end{array}$ & $\begin{array}{l}114 \pm \\
25^{a}\end{array}$ & $\begin{array}{l}132 \pm \\
25^{a}\end{array}$ & $\begin{array}{l}68 \pm 10 \\
a\end{array}$ & $\begin{array}{l}88 \pm 12 \\
a\end{array}$ & $14 \pm 4^{a}$ & $17 \pm 5^{a}$ & $889 \pm 389$ \\
\hline \multirow{6}{*}{$\begin{array}{l}\text { Nonresponders } \\
(n=11)\end{array}$} & Baseline & $\begin{array}{l}8.0 \pm 1.8 \\
b\end{array}$ & $\begin{array}{l}70 \pm \\
17\end{array}$ & $\begin{array}{l}116 \pm \\
22\end{array}$ & $\begin{array}{l}111 \pm \\
15\end{array}$ & $54 \pm 4^{b}$ & $75 \pm 5^{b}$ & $12 \pm 5$ & $14 \pm 4$ & $733 \pm 426^{b}$ \\
\hline & 100 & $8.1 \pm 2.0$ & $\begin{array}{l}74 \pm \\
23\end{array}$ & $\begin{array}{l}114 \pm \\
22\end{array}$ & $\begin{array}{l}116 \pm \\
20\end{array}$ & $61 \pm 6$ & $78 \pm 7^{a}$ & $13 \pm 5$ & $15 \pm 5$ & $666 \pm 188^{b}$ \\
\hline & 200 & $7.8 \pm 2.1$ & $\begin{array}{l}72 \pm \\
21\end{array}$ & $\begin{array}{l}114 \pm \\
21^{a}\end{array}$ & $\begin{array}{l}119 \pm \\
21\end{array}$ & $61 \pm 6^{a}$ & $79 \pm 8^{a}$ & $13 \pm 5^{a}$ & $16 \pm 5^{a}$ & $703 \pm 212^{b}$ \\
\hline & 300 & $8.0 \pm 1.8$ & $\begin{array}{l}73 \pm \\
20\end{array}$ & $\begin{array}{l}114 \pm \\
22^{a}\end{array}$ & $\begin{array}{l}122 \pm \\
22^{a}\end{array}$ & $62 \pm 8^{a}$ & $80 \pm 9^{a}$ & $14 \pm 5^{a}$ & $17 \pm 4^{\mathrm{a}}$ & $704 \pm 208^{b}$ \\
\hline & 400 & $8.0 \pm 2.0$ & $\begin{array}{l}72 \pm \\
21\end{array}$ & $\begin{array}{l}114 \pm \\
22^{a}\end{array}$ & $\begin{array}{l}121 \pm \\
25\end{array}$ & $63 \pm 8^{a}$ & $\begin{array}{l}82 \pm 10 \\
a\end{array}$ & $15 \pm 5^{a}$ & $18 \pm 4^{a}$ & $702 \pm 220^{b}$ \\
\hline & 500 & $7.9 \pm 1.9$ & $\begin{array}{l}71 \pm \\
21\end{array}$ & $\begin{array}{l}113 \pm \\
22^{a}\end{array}$ & $\begin{array}{l}125 \pm \\
25^{a}\end{array}$ & $63 \pm 8^{a}$ & $\begin{array}{l}82 \pm 10 \\
a\end{array}$ & $15 \pm 5^{a}$ & $18 \pm 4^{a}$ & $720 \pm 223$ \\
\hline Group & & $\Delta \mathrm{CO}$ & $\Delta \mathrm{SV}$ & $\Delta \mathrm{HR}$ & $\triangle S A P$ & $\triangle \mathrm{DAP}$ & $\Delta \mathrm{MAP}$ & $\Delta \mathrm{CVP}$ & $\triangle \mathrm{PAWP}$ & \\
\hline
\end{tabular}

\begin{tabular}{|c|c|c|c|c|c|c|c|c|c|}
\hline & & (\%) & $(\mathrm{mL})$ & (bpm) & $(\mathrm{mmHg})$ & $(\mathrm{mmHg})$ & $(\mathrm{mmHg})$ & $(\mathrm{mmHg})$ & $(\mathrm{mmHg})$ \\
\hline \multirow{4}{*}{$\begin{array}{l}\text { Responders } \\
(n=36)\end{array}$} & $\begin{array}{l}100- \\
\text { Baseline }\end{array}$ & $5.2 \pm 5.8$ & $\begin{array}{l}3.1 \pm \\
3.5\end{array}$ & $-1 \pm 4$ & $6 \pm 10$ & $1 \pm 5$ & $3 \pm 6$ & $1 \pm 1$ & $1 \pm 2$ \\
\hline & $\begin{array}{l}200- \\
\text { Baseline }\end{array}$ & $\begin{array}{l}11.5 \pm \\
7.1^{\mathrm{ab}}\end{array}$ & $\begin{array}{l}7.9 \pm \\
6.8^{a b}\end{array}$ & $-3 \pm 5$ & $6 \pm 19$ & $2 \pm 5^{a}$ & $4 \pm 7^{a}$ & $2 \pm 2^{a}$ & $2 \pm 2^{a}$ \\
\hline & $\begin{array}{l}300- \\
\text { Baseline }\end{array}$ & $\begin{array}{l}15.8 \pm \\
7.3^{a b}\end{array}$ & $\begin{array}{l}10.8 \pm \\
6.6^{a b}\end{array}$ & $-4 \pm 5^{a}$ & $\begin{array}{l}12 \pm 16 \\
a\end{array}$ & $3 \pm 8^{a}$ & $6 \pm 10^{a}$ & $3 \pm 2^{a}$ & $3 \pm 2^{a}$ \\
\hline & $\begin{array}{l}400- \\
\text { Baseline }\end{array}$ & $\begin{array}{l}20.7 \pm \\
9.8^{a b}\end{array}$ & $\begin{array}{l}11.9 \pm \\
5.9 \mathrm{ab}\end{array}$ & $-3 \pm 6^{a}$ & $\begin{array}{l}14 \pm 16 \\
a\end{array}$ & $4 \pm 7^{a}$ & $7 \pm 10^{a}$ & $3 \pm 2^{a}$ & $3 \pm 3^{a}$ \\
\hline
\end{tabular}




\begin{tabular}{|c|c|c|c|c|c|c|c|c|c|c|}
\hline Group & $\begin{array}{l}\text { Infusion } \\
(\mathrm{mL})\end{array}$ & $\begin{array}{l}\text { CO } \\
(\mathrm{L} / \mathrm{min})\end{array}$ & $\begin{array}{l}\text { SV } \\
(\mathrm{mL})\end{array}$ & $\begin{array}{l}\text { HR } \\
\text { (bpm) }\end{array}$ & $\begin{array}{l}\text { SAP } \\
(\mathrm{mmHg})\end{array}$ & $\begin{array}{l}\text { DAP } \\
(\mathrm{mmHg})\end{array}$ & $\begin{array}{l}\text { MAP } \\
(\mathrm{mmHg})\end{array}$ & $\begin{array}{l}\text { CVP } \\
(\mathrm{mmHg})\end{array}$ & $\begin{array}{l}\text { PAWP } \\
(\mathrm{mmHg})\end{array}$ & $\begin{array}{l}\text { SVR } \\
\text { (dynes } \cdot \mathrm{s}^{\mathrm{cm}}{ }^{-} \\
\text {5) }\end{array}$ \\
\hline & $\begin{array}{l}500- \\
\text { Baseline }\end{array}$ & $\begin{array}{l}23.2 \pm \\
13.8^{\mathrm{a} b}\end{array}$ & $\begin{array}{l}14.3 \pm \\
6.7^{\mathrm{ab}}\end{array}$ & $-3 \pm 6^{a}$ & $\begin{array}{l}15 \pm 17 \\
\mathrm{a}\end{array}$ & $4 \pm 7^{a}$ & $8 \pm 10^{a}$ & $3 \pm 2^{a}$ & $3 \pm 3^{a}$ & \\
\hline \multirow{5}{*}{$\begin{array}{l}\text { Nonresponders } \\
(n=11)\end{array}$} & $\begin{array}{l}100- \\
\text { Baseline }\end{array}$ & $1.3 \pm 7.6$ & $\begin{array}{l}3.5 \pm \\
7.6\end{array}$ & $-2 \pm 6$ & $5 \pm 10$ & $3 \pm 4$ & $3 \pm 4$ & $1 \pm 2$ & $1 \pm 1$ & \\
\hline & $\begin{array}{l}200- \\
\text { Baseline }\end{array}$ & $\begin{array}{l}-3.2 \pm 8.7 \\
\mathrm{~b}\end{array}$ & $\begin{array}{l}1.5 \pm \\
7.4^{b}\end{array}$ & $-3 \pm 7$ & $8 \pm 12$ & $3 \pm 4$ & $4 \pm 6$ & $1 \pm 1$ & $2 \pm 2^{a}$ & \\
\hline & $\begin{array}{l}300- \\
\text { Baseline }\end{array}$ & $\begin{array}{l}0.4 \pm 6.2 \\
\mathrm{~b}\end{array}$ & $\begin{array}{l}2.4 \pm \\
3.8^{b}\end{array}$ & $-3 \pm 6$ & $\begin{array}{l}11 \pm 14 \\
\mathrm{a}\end{array}$ & $4 \pm 5$ & $6 \pm 6$ & $2 \pm 2$ & $3 \pm 2^{a}$ & \\
\hline & $\begin{array}{l}400- \\
\text { Baseline }\end{array}$ & $\begin{array}{l}0.2 \pm 6.8 \\
\mathrm{~b}\end{array}$ & $\begin{array}{l}2.3^{ \pm} \\
6.3^{b}\end{array}$ & $-3 \pm 7$ & $10 \pm 17$ & $5 \pm 5^{a}$ & $7 \pm 7^{a}$ & $3 \pm 3^{a}$ & $3 \pm 1^{a}$ & \\
\hline & $\begin{array}{l}500- \\
\text { Baseline }\end{array}$ & $\begin{array}{l}-1.9 \pm 8.6 \\
b\end{array}$ & $\begin{array}{l}1.3 \pm \\
5.9^{b}\end{array}$ & $-3 \pm 8$ & $\begin{array}{l}15 \pm 18 \\
a\end{array}$ & $5 \pm 5$ & $8 \pm 8^{a}$ & $3 \pm 3^{a}$ & $3 \pm 2^{a}$ & \\
\hline
\end{tabular}

${ }^{\text {a }} P<0.05$ between baseline and volume expansion from $100 \mathrm{~mL}$ to $500 \mathrm{~mL}$ in subgroup; ${ }^{b} P<0.05$ between responders and nonresponders.

\section{Physiological variables change with mini-fluid challenge}

Baseline and changes on physiological variables are explained in detail in the Table 5 and Table 6. In R and NR, the baseline $\mathrm{ScvO}_{2}$ was 71.2 $\pm 10.7 \%$ and $72.5 \pm 11.5 \%$, and the baseline $\mathrm{SvO}_{2}$ was $68.2 \pm 9.0 \%$ and $70.7 \pm 10 \%$. After mini-fluid challenge, $\mathrm{ScvO}_{2}$ and $\mathrm{SvO}_{2}$ gradually increased. There were no significant differences between the $\mathrm{R}$ and NR. $\mathrm{Pcv}-\mathrm{aCO}_{2}$ at baseline in $\mathrm{R}$ and $\mathrm{NR}$ were $4 \mathrm{mmHg}(3-6)$ versus $5 \mathrm{mmHg}(4-6)$, and there were no significant differences between R and NR. PcV-aCO 2 did not change significantly with any amount of minifluid challenge. $\triangle \mathrm{DO}_{2}$ I increased from $13 \pm 32\left(\mathrm{~mL} / \mathrm{min} / \mathrm{m}^{2}\right)$ to $46 \pm 41\left(\mathrm{~mL} / \mathrm{min} / \mathrm{m}^{2}\right)$ in $\mathrm{R}$, while decreasing from $-13 \pm 45\left(\mathrm{~mL} / \mathrm{min} / \mathrm{m}^{2}\right)$ to $-56 \pm 53\left(\mathrm{~mL} / \mathrm{min} / \mathrm{m}^{2}\right)$ in NR. There were significant differences between the R and NR $(p<0.05)$. 
Table 5

Hemoglobin and physiological variables before and after fluid challenge in R and NR

\begin{tabular}{|c|c|c|c|c|c|c|c|c|c|}
\hline Group & $\begin{array}{l}\text { Infusion } \\
(\mathrm{mL})\end{array}$ & $\begin{array}{l}\text { HBG } \\
(\mathrm{g} / \mathrm{dL})\end{array}$ & $\begin{array}{l}\text { Hct } \\
(\%)\end{array}$ & $\begin{array}{l}\mathrm{ScvO}_{2} \\
(\%)\end{array}$ & $\begin{array}{l}\mathrm{SvO}_{2} \\
(\%)\end{array}$ & $\begin{array}{l}\mathrm{DO}_{2} \mathrm{I} \\
\left(\mathrm{mL} / \mathrm{min} / \mathrm{m}^{2}\right)\end{array}$ & $\begin{array}{l}\mathrm{VO}_{2} \mathrm{I} \\
\left(\mathrm{mL} / \mathrm{min} / \mathrm{m}^{2}\right)\end{array}$ & $\begin{array}{l}\text { Pcv- } \\
\mathrm{aCO}_{2} \\
(\mathrm{mmHg})\end{array}$ & $\begin{array}{l}\text { Pv-aCO } \\
(\mathrm{mmHg})\end{array}$ \\
\hline \multirow{6}{*}{$\begin{array}{l}\text { Responders } \\
(n=36)\end{array}$} & Baseline & $9.5 \pm 2.6$ & $26 \pm 9$ & $71.2 \pm 10.7$ & $68.2 \pm 9.0$ & $412 \pm 144$ & $103 \pm 39$ & $4(3-6)$ & $5(3-6)$ \\
\hline & 100 & $\begin{array}{l}9.3 \pm 2.5 \\
a\end{array}$ & $\begin{array}{l}25 \pm 8 \\
a\end{array}$ & $72.1 \pm 9.4$ & $68.5 \pm 8.2$ & $425 \pm 154 a b$ & $102 \pm 39$ & $5(3-6)$ & $5(3-6)$ \\
\hline & 200 & $9 \pm 2.4^{a}$ & $\begin{array}{l}24 \pm 8 \\
a\end{array}$ & $72.0 \pm 9.6$ & $69.5 \pm 7.9$ & $439 \pm 156^{a b}$ & $106 \pm 39$ & $4(2-7)$ & $4(3-6)$ \\
\hline & 300 & $\begin{array}{l}8.8 \pm 2.3 \\
a\end{array}$ & $\begin{array}{l}23 \pm 8 \\
a\end{array}$ & $73.6 \pm 9.0^{a}$ & $\begin{array}{l}71.1 \pm 7.9 \\
\mathrm{a}\end{array}$ & $445 \pm 149$ a b & $102 \pm 36$ & $5(2-6)$ & $4(3-6)$ \\
\hline & 400 & $\begin{array}{l}8.7 \pm 2.4 \\
a\end{array}$ & $\begin{array}{l}23 \pm 8 \\
a\end{array}$ & $74.3 \pm 9.3^{a}$ & $72 \pm 7.6^{a}$ & $448 \pm 157^{a b}$ & $99 \pm 38$ & $4(2-7)$ & $4(3-5)$ \\
\hline & 500 & $\begin{array}{l}8.5 \pm 2.2 \\
a\end{array}$ & $\begin{array}{l}23 \pm 7 \\
a\end{array}$ & $72.7 \pm 10.7$ & $\begin{array}{l}70.8 \pm 8.7 \\
\mathrm{a}\end{array}$ & $458 \pm 147^{a b}$ & $107 \pm 39$ & $5(2-5)$ & $4(2-5)$ \\
\hline \multirow{6}{*}{$\begin{array}{l}\text { Nonresponders } \\
(n=11)\end{array}$} & Baseline & $9.5 \pm 2.6$ & $27 \pm 7$ & $72.5 \pm 11.5$ & $70.7 \pm 10$ & $535 \pm 203$ & $120 \pm 30$ & $5(4-6)$ & $5(4-7)$ \\
\hline & 100 & $9.1 \pm 2.5^{\mathrm{a}}$ & $\begin{array}{l}25 \pm 7 \\
a\end{array}$ & $72.8 \pm 10.5$ & $71.9 \pm 9.6$ & $522 \pm 199$ a b & $119 \pm 34^{a}$ & $5(3-6)$ & $4(3-6)$ \\
\hline & 200 & $8.9 \pm 2.5^{a}$ & $\begin{array}{l}25 \pm 7 \\
a\end{array}$ & $75.1 \pm 11.0$ & $\begin{array}{l}73.4 \pm \\
10.1\end{array}$ & $488 \pm 178$ a b & $101 \pm 38^{a}$ & $5(3-6)$ & $4(4-5)$ \\
\hline & 300 & $8.6 \pm 2.4^{a}$ & $\begin{array}{l}23 \pm 6 \\
a\end{array}$ & $\begin{array}{l}75.4 \pm \\
10.5^{\mathrm{a}}\end{array}$ & $\begin{array}{l}74.2 \pm \\
10.1\end{array}$ & $487 \pm 170 a b$ & $102 \pm 36^{a}$ & $5(4-5)$ & $5(4-6)$ \\
\hline & 400 & $8.6 \pm 2.5^{a}$ & $\begin{array}{l}24 \pm 7 \\
a\end{array}$ & $75.1 \pm 11.5$ & $\begin{array}{l}73.3 \pm \\
11.1\end{array}$ & $486 \pm 183^{a b}$ & $100 \pm 37^{a}$ & $5(3-6)$ & $4(3-5)$ \\
\hline & 500 & $8.7 \pm 2.3^{a}$ & $\begin{array}{l}23 \pm 6 \\
a\end{array}$ & $74.8 \pm 11.3$ & $\begin{array}{l}71.8 \pm \\
10.4\end{array}$ & $478 \pm 179 a b$ & $99 \pm 37^{a}$ & $5(4-5)$ & $5(4-6)$ \\
\hline
\end{tabular}

Abbreviations: $\mathrm{HBG}$, hemoglobin; $\mathrm{Hct}$, hemotocrit; $\mathrm{ScvO}_{2}$, central venous oxygen saturation; $\mathrm{SvO}_{2}$, mixed venous oxygen saturation; $\mathrm{DO}_{2} \mathrm{l}$, Oxygen delivery index; $\mathrm{VO}_{2} \mathrm{l}$, oxygen consumption index; $\mathrm{Pcv}_{-\mathrm{aCO}}$, central venous-to-arterial carbon dioxide partial pressure; $\mathrm{Pv}$-aCO mixed venous-to-arterial carbon dioxide partial pressure.

${ }^{\text {a }} P<0.05$ between baseline and volune expansion from $100 \mathrm{~mL}$ to $500 \mathrm{~mL}$ in subgroup; ${ }^{\mathrm{b}} P<0.05$ between responders and nonresponders. 
Table 6

Changes in hemoglobin and physiological variables between $\mathrm{R}$ and NR

\begin{tabular}{|c|c|c|c|c|c|c|c|c|c|}
\hline \multicolumn{2}{|l|}{ Group } & $\begin{array}{l}\triangle \mathrm{HBG} \\
(\mathrm{g} / \mathrm{dL})\end{array}$ & $\begin{array}{l}\Delta \mathrm{Hct} \\
(\%)\end{array}$ & $\begin{array}{l}\Delta \mathrm{ScvO}_{2} \\
(\%)\end{array}$ & $\begin{array}{l}\Delta \mathrm{SvO}_{2} \\
(\%)\end{array}$ & $\begin{array}{l}\triangle \mathrm{DO}_{2} \mathrm{I} \\
\left(\mathrm{mL} / \mathrm{min} / \mathrm{m}^{2}\right)\end{array}$ & $\begin{array}{l}\Delta \mathrm{VO}_{2} \mathrm{I} \\
\left(\mathrm{mL} / \mathrm{min} / \mathrm{m}^{2}\right)\end{array}$ & $\begin{array}{l}\triangle \mathrm{PcV}- \\
\mathrm{aCO}_{2} \\
(\mathrm{mmHg})\end{array}$ & $\begin{array}{l}\triangle \mathrm{PV}- \\
\mathrm{aCO}_{2} \\
(\mathrm{mmHg})\end{array}$ \\
\hline \multirow{5}{*}{$\begin{array}{l}\text { Responders } \\
(n=36)\end{array}$} & $\begin{array}{l}100- \\
\text { Baseline }\end{array}$ & $-0.2 \pm 0.5$ & $-1 \pm 1$ & $0.9 \pm 4.8$ & $0.2 \pm 4.5$ & $13 \pm 32^{b}$ & $-2 \pm 22$ & $0(-2-1)$ & $0(-2-1)$ \\
\hline & $\begin{array}{l}200- \\
\text { Baseline }\end{array}$ & $\begin{array}{l}-0.5 \pm 0.5 \\
a\end{array}$ & $\begin{array}{l}-2 \pm 2 \\
a\end{array}$ & $0.8 \pm 6.0$ & $\begin{array}{l}1.3 \pm 4.8 \\
a\end{array}$ & $27 \pm 38$ ab & $3 \pm 23^{b}$ & $-1(-2-2)$ & $0(-3-1)$ \\
\hline & $\begin{array}{l}300- \\
\text { Baseline }\end{array}$ & $\begin{array}{l}-0.7 \pm 0.5 \\
a\end{array}$ & $\begin{array}{l}-3 \pm 2 \\
a\end{array}$ & $\begin{array}{l}2.4 \pm \\
6.9^{a}\end{array}$ & $\begin{array}{l}2.9 \pm 5.5 \\
a\end{array}$ & $33 \pm 30$ a b & $-1 \pm 28$ & $0(-1-2)$ & $-1(-2-1)$ \\
\hline & $\begin{array}{l}400- \\
\text { Baseline }\end{array}$ & $\begin{array}{l}-0.9 \pm 0.6 \\
a\end{array}$ & $\begin{array}{l}-3 \pm 2 \\
a\end{array}$ & $\begin{array}{l}3.0 \pm \\
8.8^{a}\end{array}$ & $\begin{array}{l}3.8 \pm 5.3 \\
a\end{array}$ & $36 \pm 38$ a b & $-4 \pm 33$ & $0(-2-2)$ & $0(-2-1)$ \\
\hline & $\begin{array}{l}500- \\
\text { Baseline }\end{array}$ & $\begin{array}{l}-1.0 \pm 0.6 \\
a\end{array}$ & $\begin{array}{l}-3 \pm 2 \\
a\end{array}$ & $1.5 \pm 6.5$ & $\begin{array}{l}2.6 \pm 6.2 \\
a\end{array}$ & $46 \pm 41^{a b}$ & $5 \pm 27^{b}$ & $0(-2-1)$ & $-1(-3-1)$ \\
\hline \multirow{5}{*}{$\begin{array}{l}\text { Nonresponders } \\
(n=11)\end{array}$} & $\begin{array}{l}\text { 100- } \\
\text { Baseline }\end{array}$ & $-0.4 \pm 0.5$ & $-1 \pm 2$ & $0.3 \pm 2.6$ & $1.2 \pm 4.2$ & $-13 \pm 45^{b}$ & $-6 \pm 14$ & $0(-1-0)$ & $-1(-3-1)$ \\
\hline & $\begin{array}{l}200- \\
\text { Baseline }\end{array}$ & $-0.6 \pm 0.6$ & $-2 \pm 2$ & $2.6 \pm 5.7$ & $2.7 \pm 4.4$ & $-47 \pm 52^{a b}$ & $-19 \pm 21^{a b}$ & $0(-3-1)$ & $-1(-2-0)$ \\
\hline & $\begin{array}{l}300- \\
\text { Baseline }\end{array}$ & $\begin{array}{l}-0.9 \pm 0.6 \\
a\end{array}$ & $\begin{array}{l}-3 \pm 2 \\
a\end{array}$ & $\begin{array}{l}2.9 \pm \\
4.1^{a}\end{array}$ & $3.5 \pm 5.9$ & $-49 \pm 36$ a b & $-18 \pm 16^{a}$ & $0(-2-1)$ & $0(-2-1)$ \\
\hline & $\begin{array}{l}400- \\
\text { Baseline }\end{array}$ & $\begin{array}{l}-0.8 \pm 0.5 \\
a\end{array}$ & $\begin{array}{l}-3 \pm 2 \\
a\end{array}$ & $2.6 \pm 5.8$ & $2.6 \pm 7.4$ & $-48 \pm 36$ a b & $-19 \pm 23^{a}$ & $1(-2-1)$ & $-1(-3-1)$ \\
\hline & $\begin{array}{l}500- \\
\text { Baseline }\end{array}$ & $\begin{array}{l}-0.8 \pm 0.7 \\
a\end{array}$ & $\begin{array}{l}-3 \pm 3 \\
a\end{array}$ & $2.3 \pm 5.1$ & $1.1 \pm 4.5$ & $-56 \pm 53$ a b & $-21 \pm 28$ a b & $-1(-2-1)$ & $0(-3-1)$ \\
\hline
\end{tabular}

a $P<0.05$ between baseline and volume expansion from $100 \mathrm{~mL}$ to $500 \mathrm{~mL}$ in subgroup; ${ }^{\mathrm{b}} P<0.05$ between responders and nonresponders.

\section{Predictive value of mini-fluid challenge}

The correlation between $\Delta \mathrm{CO}_{100 \mathrm{~mL}}$ and $\Delta \mathrm{CO}_{500 \mathrm{~mL}}$ was negative $(\mathrm{r}=0.26, p=0.08)$, whereas $\Delta \mathrm{CO}_{200 \mathrm{~mL}}$ and $\Delta \mathrm{CO}_{500 \mathrm{~mL}}, \Delta \mathrm{CO}_{300 \mathrm{~mL}}$ and $\Delta \mathrm{CO}_{500 \mathrm{~mL}}, \Delta \mathrm{CO}_{400 \mathrm{~mL}}$ and $\Delta \mathrm{CO}_{500 \mathrm{~mL}}$ were all positive $(\mathrm{r}=0.57, \mathrm{r}=0.73, \mathrm{r}=92 ; p<0.05)$ respectively. ROC of $\Delta \mathrm{CO} \%$ after every $100 \mathrm{~mL}$ fluid bolus were analyzed for predicting FR (Fig. 5). The AUROC were calculated and compared separately (Table 7). In this study, a mini fluid of $100 \mathrm{~mL}$ had a poor predictive ability (AUC $=0.67, p>0.05) . \Delta \mathrm{CO}_{200 \mathrm{~mL}}$ demonstrated a predictive value for $\mathrm{FR}$ with an $\mathrm{AUC}$ of $0.93(95 \% \mathrm{Cl}$ : $0.84-1.00, p<0.05)$. The best cutoff value of $\Delta \mathrm{CO}_{200 \mathrm{~mL}}$ was $1.9 \%$, which was lower than the reproducibility of any kinds of $\mathrm{CO}$ measurements (sensitivity of $97.2 \%$ and specificity of $81.8 \%$ ). Taking reproducibility and reliability into account, the best cutoff value was $5.2 \%$, with a sensitivity of $83.3 \%$, and a specificity of $90.9 \%$. The AUC of $\Delta \mathrm{CO}_{300 \mathrm{~mL}}$ and $\Delta \mathrm{CO}_{400 \mathrm{~mL}}$ were 0.95 and 0.97 (p<0.05) There was no difference between the predictive abilities between the $\Delta \mathrm{CO}_{200 \mathrm{~mL}}, \Delta \mathrm{CO}_{300 \mathrm{~mL}}$ and $\Delta \mathrm{CO}_{400 \mathrm{~mL}}$ compared with the $\Delta \mathrm{CO}_{500 \mathrm{~mL}}$. 
Table 7

The predictive characteristics of the increase in CO in mini-fluid challenge for a response of $\mathrm{CO}>10 \%$ over $500 \mathrm{~mL}$ of fluid infusion

\begin{tabular}{|c|c|c|c|c|c|c|c|c|c|c|c|}
\hline $\begin{array}{l}\text { Fluid } \\
\text { bloused }\end{array}$ & AUC & $P$ & $95 \% \mathrm{Cl}$ & $\begin{array}{l}\text { Cut off } \\
\text { value, } \%\end{array}$ & $\begin{array}{l}\text { Sensitivity } \\
(\%)\end{array}$ & $\begin{array}{l}\text { Specificity } \\
(\%)\end{array}$ & $Y$ & $\begin{array}{l}\text { Difference between } \\
\text { areas }\end{array}$ & $P$ & $r_{s}$ & $P\left(r_{s}\right)$ \\
\hline 100 & 0.67 & 0.11 & $\begin{array}{l}0.52- \\
0.80\end{array}$ & NA & NA & NA & NA & 0.33 & $\begin{array}{l}<.05 \\
0.05\end{array}$ & 0.26 & 0.08 \\
\hline 200 & 0.93 & $\begin{array}{l}< \\
0.05\end{array}$ & $\begin{array}{l}0.82- \\
0.99\end{array}$ & 1.9 & 97.2 & 81.8 & 0.79 & 0.06 & 0.16 & 0.57 & $\begin{array}{l}< \\
0.05\end{array}$ \\
\hline 300 & 0.96 & $\begin{array}{l}<.05 \\
0.05\end{array}$ & $\begin{array}{l}0.85- \\
0.99\end{array}$ & 4.8 & 100 & 90.9 & 0.91 & 0.04 & 0.32 & 0.73 & $\begin{array}{l}< \\
0.05\end{array}$ \\
\hline 400 & 0.97 & $\begin{array}{l}<.05 \\
0.05\end{array}$ & $\begin{array}{l}0.87- \\
1.000\end{array}$ & 6.2 & 97.2 & 90.9 & 0.88 & 0.03 & 0.20 & 0.92 & $\begin{array}{l}< \\
0.05\end{array}$ \\
\hline
\end{tabular}

Abbreviations: AUC, area under the curve; $\mathrm{Cl}$, confidence interval; $Y$ Youden index; $\mathrm{r}_{\mathrm{s}}$, Spearman's correlation.

MAP $<61 \mathrm{mmHg}$ at baseline was able to predict a fluid response (AUC $=0.71,95 \% \mathrm{Cl} p<0.05)$ with a sensitivity and specificity of $100 \%$ and 100\% (Fig. 6).

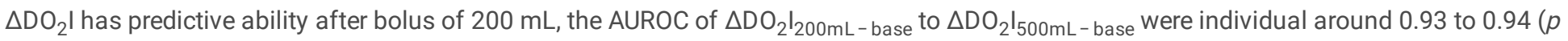
$<0.05$ ), and the cut-off was around 2.5-3.5\% (Fig. 7,8, Table 8)

Table 8

The predictive characteristics of the increase in $\triangle \mathrm{DO}_{2} \mathrm{l}$ in mini-fluid challenge for a response of $\mathrm{CO}>10 \%$ over $500 \mathrm{~mL}$ of fluid infusion

\begin{tabular}{|c|c|c|c|c|c|c|c|c|c|}
\hline & \multicolumn{2}{|c|}{ Mean \pm SD } & \multirow[t]{2}{*}{$\mathbf{P}$} & \multirow[t]{2}{*}{ AUC } & \multirow[t]{2}{*}{$\mathbf{P}$} & \multirow[t]{2}{*}{$95 \% \mathrm{Cl}$} & \multirow{2}{*}{$\begin{array}{l}\text { Cut-off } \\
(\mathrm{g} / \mathrm{L})\end{array}$} & \multirow[t]{2}{*}{ Sensitivity(\%) } & \multirow[t]{2}{*}{ Specificity(\%) } \\
\hline & $\begin{array}{l}R \\
(n=35)\end{array}$ & $\begin{array}{l}\text { NR } \\
(n=11)\end{array}$ & & & & & & & \\
\hline $\begin{array}{l}\triangle \mathrm{DO}_{2} \mathrm{I} \\
100 \mathrm{~mL} \text {-base }\left(\mathrm{mL} / \mathrm{min} / \mathrm{m}^{2}\right)\end{array}$ & $13 \pm 32$ & $-13 \pm 45$ & $<0.05$ & 0.68 & 0.07 & $0.49-0.87$ & NA & NA & NA \\
\hline $\begin{array}{l}\triangle \mathrm{DO}_{2} \mathrm{I} \\
200 \mathrm{~mL} \text {-base }\left(\mathrm{mL} / \mathrm{min} / \mathrm{m}^{2}\right)\end{array}$ & $27 \pm 38^{a}$ & $-47 \pm 52^{a}$ & $<0.05$ & 0.94 & $<0.05$ & 0.88-1.0 & 2.5 & 71.4 & 100 \\
\hline $\begin{array}{l}\triangle \mathrm{DO}_{2} \mathrm{I} \\
300 \mathrm{~mL}-\text { base }\left(\mathrm{mL} / \mathrm{min} / \mathrm{m}^{2}\right)\end{array}$ & $33 \pm 30^{a}$ & $-49 \pm 36^{a}$ & $<0.05$ & 0.93 & $<0.05$ & $0.80-1.0$ & 3.5 & 82.9 & 90.0 \\
\hline $\begin{array}{l}\triangle \mathrm{DO}_{2} \mathrm{I} \\
400 \mathrm{~mL}-\text { base }\left(\mathrm{mL} / \mathrm{min} / \mathrm{m}^{2}\right)\end{array}$ & $36 \pm 38^{a}$ & $-48 \pm 36^{a}$ & $<0.05$ & 0.97 & $<0.05$ & 0.94-1.0 & 3.5 & 85.7 & 100 \\
\hline $\begin{array}{l}\triangle \mathrm{DO}_{2} \mathrm{I} \\
500 \mathrm{~mL}-\text { base }\left(\mathrm{mL} / \mathrm{min} / \mathrm{m}^{2}\right)\end{array}$ & $46 \pm 41^{a}$ & $-56 \pm 53^{a}$ & $<0.05$ & 0.94 & $<0.05$ & $0.83-1.0$ & 2.5 & 82.9 & 90.9 \\
\hline Abbreviation: $\mathrm{DO}_{2} \mathrm{I}, \mathrm{Oxy}$ & lelivery i & & & & & & & & \\
\hline
\end{tabular}

$\mathrm{ScvO}_{2}, \mathrm{SvO}_{2}, \mathrm{Pcv}-\mathrm{aCO}_{2}, \Delta \mathrm{ScvO}_{2}, \Delta \mathrm{SvO}_{2}, \Delta \mathrm{Pcv}_{-\mathrm{aCO}_{2}}$ were not able to predict a positive fluid response with any amount of mini-fluid challenge in this study (Table 9). 
Table 9

Relationship between baseline and change after $500 \mathrm{~mL} \mathrm{FC} \mathrm{ScvO}, \mathrm{SvO}_{2}, \mathrm{Pcv}-\mathrm{aCO} 2 \mathrm{and} \mathrm{Pv}$ $\mathrm{aCO}_{2}$ levels and changes in cardiac output $(\triangle \mathrm{CO} \%)$ in all patients and the AUC of each

indicators

\begin{tabular}{|c|c|c|c|c|c|}
\hline & $r_{s}$ & $P\left(r_{s}\right)$ & AUC & $P$ & $95 \% \mathrm{Cl}$ \\
\hline ScvO2 & -0.18 & 0.22 & 0.46 & 0.65 & $0.26-0.65$ \\
\hline Sv02 & -0.33 & $<0.05$ & 0.45 & 0.63 & $0.26-0.65$ \\
\hline Pcv-aCO2 & 0.07 & 0.67 & 0.43 & 0.48 & $0.25-0.61$ \\
\hline Pv-aCO2 & 0.22 & 0.14 & 0.43 & 0.48 & $0.25-0.61$ \\
\hline$\Delta \mathrm{ScvO}_{2}(500$-base $)$ & -0.04 & 0.69 & 0.48 & 0.85 & $0.29-0.68$ \\
\hline$\Delta \mathrm{SvO}_{2}(500$-base) & 0.27 & 0.08 & 0.56 & 0.59 & $0.37-0.74$ \\
\hline$\Delta$ Pcv-aCO 2 (500-base) & -0.06 & 0.80 & 0.51 & 0.94 & $0.32-0.69$ \\
\hline$\Delta \mathrm{PV}-\mathrm{aCO}_{2}(500$-base $)$ & 0.26 & 0.07 & 0.57 & 0.49 & $0.37-0.77$ \\
\hline
\end{tabular}

Abbreviations: AUC, area under the curve; $\mathrm{Cl}$, confidence interval; $r_{\mathrm{s}}$, Spearman's correlation.

\section{Discussion}

Optimizing CO by increasing preload is based on the assumption that increased blood volume may subsequently promote stroke volume (SV) as described by the Frank-Starling law. Predicting fluid responsiveness is to test whether the patient was in the ascending part of the Frank-Starling curve (preload dependence state) and may benefit from fluid treatment[24, 27-30]. Although numerous measurements can predict FR, the fluid challenge is a gold standard for assessing FR and is widely used. The new concept of mini-fluid challenge is to use a minimun amount of fluid to avoid overload risk, whereas a median volume of $500 \mathrm{~mL}$ has been used before.[14, 31, 32]. A metanalysis demonstrated that a mini volume of $100 \mathrm{~mL}$ could predict preload responsiveness, the pooled AUC was 0.91 (95\% CI 0.85-0.97), with a pooled sensitivity of $0.82(95 \% \mathrm{Cl} 0.76-0.88)$ and specificity of $0.83(95 \% \mathrm{Cl} 0.77-0.89)[33]$. No similar findings were found in our study: the predictive power of $100 \mathrm{~mL}$ is disappointing with an AUROC of 0.69 .

The main reason for these differences may be the difference in the study population. Our study focused on septic shock patients, whose cardio-vascular system has undergone major perturbations and profound alterations to the endothelium. Capillary leakage caused by epithelial barrier dysfunction occurs, as a consequence, the intravascular volume is insufficient and unstressed volume substantially reduced[34]. The adjustment and shift between unstressed volume and stressed volume introduce a role for volume expansion that is not simply to increase $\mathrm{CO}$ but rather to ensure reserves[35]. In these patients, a larger dose may be required for an effective test, whereas $100 \mathrm{~mL}$ of fluid seemed too little to predict the FR. Among the previous 7 studies, only 1 mini-fluid study focused on septic shock. Wang's study showed a similar result to ours that the predictive power with $100 \mathrm{~mL}$ was less satisfying. In other mini-fluid challenge investigation, the study population was perioperative patients in a stable hemodynamic state. In this study, we chose colloid of gelatin to perform the fluid challenge, which can stay in vascular longer and may increase CO more than crystalloid. The marked change, however, showed in $200 \mathrm{~mL}$.

In spite of the effect of heterogeneity of study population, Aya et al[22]. and Smoerenberg et al[16]. were unable to reproduce similar results as well, who showed the predicted minimal volume is $4 \mathrm{~mL} / \mathrm{kg}$ to defined FC (CO measured by LiDCOplus monitor), or minimal volume of $150 \mathrm{~mL}$ CO measured by Modelflow ${ }^{\mathrm{R}} \mathrm{COm}$ and $200 \mathrm{~mL}$ by PulseCO ${ }^{\mathrm{R}}$ COli. These two studies implied a negative predictive value of a minimum dose of $100 \mathrm{~mL}$ to reliably assess FR even in a fairly homogeneous sample of postoperative cardiac surgery patients. It suggests that the specificity and sensitivity values of mini fluid challenge may change depending on the device used to measure the CO. To the best of our knowledge, CO measurements including thermodilution (PAC), pulse index continuous CO, Doppler echocardiography (TTE or TEE) and Fick techniques, have been most commonly used to assess FR in recent decades. In the mini fluid challenge, pulse contour CO was used in 4 out of the 7 cases, and TTE was used in 2 out of the 7 cases, while PAC, as a standard clinical reference method for CO monitoring was not used in the above studies.

However, the CO measurements by Doppler echocardiography (we have discussed only the VTi method, which is the most popular and presented in recent studies) have been validated for a long time for its noninvasive, but its accuracy is limited by the devices and technicians. In method of echocardiography, SV is affected by VTi and the aortic valve area. When estimating the changes in SV, the value of VTi is the main component of the SV calculation whereas the aortic valve area is supposed to be constant. It is well known that either the quality and

Page $12 / 22$ 
sharpness of image or the process of technician may have an influence on the value of VTi. Moreover, Gorassi et al.[36] demonstrated that pulsed Doppler has limitations in detecting high cardiac output values when the blood flow velocity is greater than $2 \mathrm{~m} / \mathrm{sm}$, especially in septic shock patients who showed the greatest variability in $\mathrm{CO}$. What is more, the CO measured by PAC integrated over several heartbeats, while the VTi was measured on a beat-to-beat basis, and then calculates CO by the products of SV and HR, which may increase the potential error of CO estimation. VTi seemed to be more likely influenced by heart-lung interaction than PAC, and previous studies have shown that CO is more reliable on an average of serial measurements[37]. Based on the published studies evidences, the accuracy of PAC is higher than that of the transthoracic echocardiography[23, 36, 38].

At the same time, the precision of new generation CO measurement, especially methods coupled with thermodilution and pulse contour analysis, such as Pulse index Continuous Cardiac Output combination (PiCCO), is also unclear, because more variables will affect the estimation of CO. Pulse contour analysis measures $\mathrm{CO}$ indirectly by integrating a variety of characteristics of the pressure waveform to calculate stroke volume. In the equation of $\mathrm{CO}=\mathrm{SV} \times \mathrm{HR}, \mathrm{SV}$ is calculated from area under systolic portion of arterial waveform trace and aortic impedance, in which the arterial pressure waveform is complex and aortic impedance varies between individuals and within individuals, especially in septic shock patients whose hemodynamic state is characterized by distributive shock and the vascular impedance is unpredictable. Although the pulse contour analysis has been calibrated by trans-cardiopulmonary thermodilution to overcome some limitations, the analysis remains potential inaccuracy due to multitude of variables in the equation of $\mathrm{CO}$ calculation. A great deal of studies and meta-analysis have been done to compare the precision of new generation measurements of CO with PAC, but why not perform a minifluid challenge in classic thermodilution by PAC.

In addition to taking the mini-volume into fluid challenge, the threshold of predictive value is also a controversial topic. In most clinical practice, and even in most studies, the definition of FR is based on the assumption that thermodilution is the only method validated to detect a 10-15\% increase in $\mathrm{CO}[23]$. This consensus was originally derived from the understanding of the sources of errors of PAC, that is, three measurements are sufficient if the CO differs by $10 \%$ or less. On the contrary, if the CO difference exceeds $10 \%$, the measurements are considered unreliable[39]. It is worth noting that these technique errors were mostly introduced by the measurement of injecting iced water

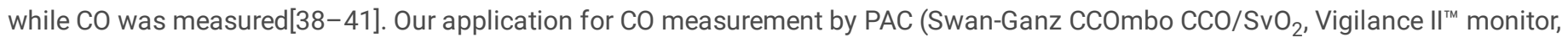
Edwards Lifesciences, Irvine, CA, USA) in this study is automatic heating, and iced water injection is no longer needed. The sources of error including temperature of iced water, volume of injection and even injection speed will not affect the CO measurement accuracy during this protocol. In order to avoid measurement errors and enhance the reproducibly of the results, we took an average CO value of 3 measurements at each point in time as a CO determination. In this study, the best cutoff value of $1.9 \%$ was an inferior threshold to the interobserver variability of PAC. Thus, considering both clinical feasibility and reproducibility, the cutoff value was $5.2 \%$, with a specificity of $83.3 \%$ and $90.9 \%$, respectively. An increase in $\mathrm{CO}$ greater than $5.2 \%$ after $200 \mathrm{~mL}$ may be more clinically relevant. The correlation between $\Delta \mathrm{CO}_{200 \mathrm{~mL}}$ and $\Delta \mathrm{CO}_{500 \mathrm{~mL}}$ suggests that the greater the increase in $\Delta \mathrm{CO}_{200 \mathrm{~mL}}$, the more we can expect a similar increase in $\Delta \mathrm{CO}_{500 \mathrm{~mL}}$. If we accepted CO > $10 \%$ in mini-FC, both the sensitivity and specificity were too low (60.7\% and $73.7 \%$ ) to predict FR, and up to $32 \%$ of R may possibly be misclassified as NR (Fig. 9).

In the design of this study, we attempted to find a surrogate of $\mathrm{CO}$, which can evaluate FR simply and practically when there is no CO monitoring available or inconvenient. $\mathrm{SvO}_{2}$ and $\mathrm{ScvO}_{2}$ are a pair of parameters wildly used in clinical practice to assess whether $\mathrm{CO}$ and oxygen delivery $\left(\mathrm{DO}_{2}\right)$ are sufficient to meet the patient's need and to guide fluid resuscitation[42]. The well-known study by Rivers et al.[43] indicated that targeting a $\mathrm{ScvO}_{2}>70 \%$ in the early stage of resuscitation may improve outcomes. In our study, both in $\mathrm{R}$ and $\mathrm{NR}$, the mean value of $\mathrm{ScvO}_{2}$ were higher than $70 \%$ while that of $\mathrm{SvO}_{2}$ were higher than $65 \%$. Neither the actual value of $\mathrm{ScvO}_{2}$ and $\mathrm{SvO}_{2}$ nor the $\Delta \mathrm{ScvO}_{2}$ and $\Delta \mathrm{SvO}_{2}$ were meaningless to predict FR. In fact, $\mathrm{ScvO}_{2}$ and $\mathrm{SvO}_{2}$ can be influenced by oxygen extraction. We confirmed the result of Velissaris et al[42] that a high level of $\mathrm{ScvO}_{2}$ and $\mathrm{SvO}_{2}$ levels did not exclude FR. In this study, there were 17 cases out of 37 (45.9\%) with $\mathrm{ScvO}_{2}$ were greater than $70 \%$, and 20 cases out of 37 (54.1\%) with $\mathrm{ScvO}_{2}$ greater than $65 \%$ in $\mathrm{FR}$. Based on the same theory, Pcv-aCO 2 are considered as alternative markers of tissue hypoperfusion and are used to guide treatment for shock. However, there was no difference between R and NR before and after FC. For the indicators we simply used in clinical practice, MAP $<61 \mathrm{mmHg}$ before FC is a strong indicator for FR, with a sensitivity and specificity of $100 \%$ and $100 \%$. Although $\Delta \mathrm{DO}_{2} \mathrm{I}$ exhibited predictive power in this study, it correlated to the changes of $\mathrm{CO}$ and could not be used as a surrogate of $\mathrm{CO}$.

Several limitations of this study need to be discussed. First, as we discussed before, the accuracy varies with different hemodynamic techniques. With the development of techniques in assessing the circulatory function, the new generation measurements noted by less or non-invasive, such as TEE, bio-impedance and TTE are more likely to be applied in recent decade[36, 44]. Our findings depend on PAC to monitor $\mathrm{CO}$, and the results may not be extrapolated to other techniques used to monitor $\mathrm{CO}$. Second, $77 \%$ of the patients responded to fluid administration after a total volume of $500 \mathrm{~mL}$ gelatin, and the proportion of responders (PR) was higher than in other studies. Our FC was completed within 40 minutes from the first bolus. Theoretically, PR decreases with a long infusion time, as described by Toscani et al.[45],

Page 13/22 
but our results are just the opposite. We compared the similar results of high PR, which appeared in Smorenberg's study, with a PR of 71\% at the end of FR. These trials shared two same points, one was that patients subsequently received a $500 \mathrm{~mL}$ volume expansion by several intravenous boluses, and the other was that both used the colloid in FC. So, a reasonable question is whether the high PR may be due to the accumulative of fluid or the colloid, which supposed to remain in the intravascular compartment longer. To answer this question, we need to further study the pharmacodynamic outcomes and its effect on different approaches of FC. Third, we did not find a meaningful surrogate of $\mathrm{CO}$ that can predict FR and guide fluid therapy. In future study, a feasible and simple clinical indicator for predicting FR needs to be found. Above all, future research may be conducted to investigate the diagnostic value of the mini-FC using crystalloids, and the accuracy grey zone to detect $\mathrm{CO}$ changing by using different $\mathrm{CO}$ monitor. Furthermore, pharmacodynamic and pathophysiology mechanisms $\mathrm{FC}$ need to be studied.

\section{Conclusions}

A mini-fluid challenge of $200 \mathrm{~mL}$ colloid can predict fluid responsiveness in septic shock patients. $\Delta \mathrm{CO} \%$ greater than $5.2 \%$ after a minivolume of $200 \mathrm{~mL} 4 \%$ gelatin is a good predictor. Further studies are needed to confirm this result and detect the predictive value of mini fluid by using crystalloids.

\section{List Of Abbreviations}

\begin{tabular}{|ll|}
\hline AUC & Area under curve \\
\hline CO & Cardiac output \\
\hline CVP & Central venous pressure \\
\hline DO $_{2}$ & Oxygen delivery \\
\hline DBP & Diastolic blood pressure \\
\hline DO $_{2}$ I & Oxygen delivery index \\
FC & Fluid challenge \\
FR & Fluid responsiveness \\
\hline HR & Heart rate \\
\hline Hb & Hemoglobin \\
ICU & Intensive care unit \\
\hline Lac & Lactate \\
\hline MAP & Mean arterial pressure \\
\hline Min & Minute \\
N & Nonresponder \\
R & Responder \\
ROC & Receiver operator characteristic \\
\hline PAC & Pulmonary artery catheter \\
Pmsf & Mean systemic filling pressure \\
PC/VC & Pressure control/Volume control \\
\hline Pcv-aCO & Central venous-to-arterial carbon dioxide partial pressure \\
\hline SV & Stroke volume \\
\hline SVR & Systemic vascular resistance \\
\hline ScvO & Central venous oxygen saturation \\
SvO & \\
\hline SBP & Mixed venous oxygen saturation \\
\hline
\end{tabular}

Page 14/22 


\section{Declarations}

\section{Ethics approval}

Ethical approval for the study was provided by the Ethical Committee of Peking Union Medical College Hospital (ZS-1085). Written informed consent was obtained from all patients or their legal representative.

\section{Consent for publication}

Not applicable.

\section{Availability of data and materials}

All data generated and/or analyzed during this study are included in this published article (and its supplementary information files).

\section{Competing interests}

The authors declare that they have no competing interests.

\section{Funding}

The study has been supported by the Ministry of Science and Technology of People's Republic of China (2020YFC0841300).

\section{Authors' contributions}

TY is the chief investigator of this study in designing and conducting the study, also participated in data acquisition and analysis, drafted the manuscript. BD is made a significant contribution to the design and supervised the analyses. LW, WJ, CYW, JMP and XYH are co-applicants and site principal investigators and made a significant contribution to conduct of the study. TY and SL are responsible for day to day study management. All authors commented critically on the manuscript and reviewed and approved the final manuscript.

\section{Acknowledgements}

We appreciate all physicians, nurses and staff of the Medical Intensive Care Unit (Peking Union Medical College and Chinese Academy of Medical Sciences) for their support and participation in this study.

\section{References}

1. Dellinger RP, Carlet JM, Masur H, Gerlach H, Calandra T, Cohen J, Gea-Banacloche J, Keh D, Marshall JC, Parker MM, et al. Surviving Sepsis Campaign guidelines for management of severe sepsis and septic shock. Crit Care Med. 2004;32(3):858-73.

2. Dellinger RP, Levy MM, Carlet JM, Bion J, Parker MM, Jaeschke R, Reinhart K, Angus DC, Brun-Buisson C, Beale R, et al. Surviving Sepsis Campaign: international guidelines for management of severe sepsis and septic shock: 2008. Crit Care Med. 2008;36(1):296-327.

3. Dellinger RP, Levy MM, Rhodes A, Annane D, Gerlach H, Opal SM, Sevransky JE, Sprung CL, Douglas IS, Jaeschke R, et al. Surviving Sepsis Campaign: international guidelines for management of severe sepsis and septic shock, 2012. Intensive Care Med.

2013;39(2):165-228.

4. Levy MM, Evans LE, Rhodes A. The Surviving Sepsis Campaign Bundle: 2018 update. Intensive Care Med. 2018;44(6):925-8.

5. Cordemans C, De Laet I, Van Regenmortel N, Schoonheydt K, Dits H, Huber W, Malbrain ML: Fluid management in critically ill patients: the role of extravascular lung water, abdominal hypertension, capillary leak, and fluid balance. Ann Intensive Care 2012, 2(Suppl 1 Diagnosis and management of intra-abdominal hyperten):S1.

6. O'Connor ME, Prowle JR. Fluid Overload. Crit Care Clin. 2015;31(4):803-21. 
7. Vaara ST, Korhonen AM, Kaukonen KM, Nisula S, Inkinen O, Hoppu S, Laurila JJ, Mildh L, Reinikainen M, Lund V, et al. Fluid overload is associated with an increased risk for 90-day mortality in critically ill patients with renal replacement therapy: data from the prospective FINNAKI study. Crit Care. 2012;16(5):R197.

8. Kelm DJ, Perrin JT, Cartin-Ceba R, Gajic O, Schenck L, Kennedy CC. Fluid overload in patients with severe sepsis and septic shock treated with early goal-directed therapy is associated with increased acute need for fluid-related medical interventions and hospital death. Shock. 2015;43(1):68-73.

9. Corl KA, Prodromou M, Merchant RC, Gareen I, Marks S, Banerjee D, Amass T, Abbasi A, Delcompare C, Palmisciano A, et al. The Restrictive IV Fluid Trial in Severe Sepsis and Septic Shock (RIFTS): A Randomized Pilot Study. Crit Care Med. 2019;47(7):951-9.

10. Silversides JA, Perner A, Malbrain M. Liberal versus restrictive fluid therapy in critically ill patients. Intensive Care Med. 2019;45(10):1440-2.

11. Sakr Y, Rubatto Birri PN, Kotfis K, Nanchal R, Shah B, Kluge S, Schroeder ME, Marshall JC, Vincent JL. Intensive Care Over Nations I: Higher Fluid Balance Increases the Risk of Death From Sepsis: Results From a Large International Audit. Crit Care Med. 2017;45(3):38694.

12. Malbrain M, Van Regenmortel N, Saugel B, De Tavernier B, Van Gaal PJ, Joannes-Boyau O, Teboul JL, Rice TW, Mythen M, Monnet X. Principles of fluid management and stewardship in septic shock: it is time to consider the four D's and the four phases of fluid therapy. Ann Intensive Care. 2018;8(1):66.

13. Ueyama H, Kiyonaka S. Predicting the Need for Fluid Therapy-Does Fluid Responsiveness Work? J Intensive Care. $2017 ; 5: 34$.

14. Shi R, Monnet X, Teboul JL. Parameters of fluid responsiveness. Curr Opin Crit Care. 2020;26(3):319-26.

15. Jozwiak M, Monnet X, Teboul JL. Prediction of fluid responsiveness in ventilated patients. Ann Transl Med. $2018 ; 6(18): 352$.

16. Smorenberg A, Cherpanath TGV, Geerts BF, de Wilde RBP, Jansen JRC, Maas JJ, Groeneveld ABJ. A mini-fluid challenge of 150 mL predicts fluid responsiveness using Modelflow(R) pulse contour cardiac output directly after cardiac surgery. J Clin Anesth. 2018;46:1722.

17. Xiao-ting W, Hua Z, Da-wei L, Hong-min Z, Huai-wu H, Yun L, Wen-zhao C. Changes in end-tidal CO2 could predict fluid responsiveness in the passive leg raising test but not in the mini-fluid challenge test: A prospective and observational study. J Crit Care. 2015;30(5):10616.

18. Biais M, de Courson H, Lanchon R, Pereira B, Bardonneau G, Griton M, Sesay M, Nouette-Gaulain K. Mini-fluid Challenge of 100 ml of Crystalloid Predicts Fluid Responsiveness in the Operating Room. Anesthesiology. 2017;127(3):450-6.

19. Yunfan Wu SZ, Zhihua Zhou, Bao, Liu. A 10-second fluid challenge guided by transthoracic echocardiography can predict fluid responsiveness. Critical Care 2014, 18.

20. Mallat J, Meddour M, Durville E, Lemyze M, Pepy F, Temime J, Vangrunderbeeck N, Tronchon L, Thevenin D, Tavernier B. Decrease in pulse pressure and stroke volume variations after mini-fluid challenge accurately predicts fluid responsivenessdagger. Br $\mathrm{J}$ Anaesth. 2015;115(3):449-56.

21. Laurent Muller MT, Bousquet P-J, Riu-Poulenc B, Louart G, Candela D. Lana Zoric,Carey Suehs, Jean-Emmanuel de La Coussaye, Nicolas Molinari, Jean-Yves Lefrant.: An increase in aortic blood flow after an infusion of $100 \mathrm{ml}$ colloid over 1 minute can predict fluid responsiveness. Anesthesiology. 2011;115:541-7.

22. Hollmann D. Aya AR, Ster IC, Fletcher N, Grounds RM. Maurizio Cecconi;: Hemodynamic Effect of Different Doses of Fluids for a Fluid Challenge A Quasi Randomized Controlled Study. Crit Care Med. 2017;45:161-8.

23. Ansari BM, Zochios V, Falter F, Klein AA. Physiological controversies and methods used to determine fluid responsiveness: a qualitative systematic review. Anaesthesia. 2016;71(1):94-105.

24. Jalil BA, Cavallazzi R. Predicting fluid responsiveness: A review of literature and a guide for the clinician. Am J Emerg Med. 2018;36(11):2093-102.

25. Vallet B, Pinsky MR, Cecconi M. Resuscitation of patients with septic shock: please "mind the gap". Intensive Care Med. 2013;39(9):1653-5.

26. Helmy TA, El-Reweny EM, Ghazy FG. Prognostic Value of Venous to Arterial Carbon Dioxide Difference during Early Resuscitation in Critically III Patients with Septic Shock. Indian J Crit Care Med. 2017;21(9):589-93.

27. Fluid responsiveness in critically ill patients.

28. Guerin L, Monnet X, Teboul JL. Monitoring volume and fluid responsiveness: from static to dynamic indicators. Best Pract Res Clin Anaesthesiol. 2013;27(2):177-85.

29. Cecconi M, Parsons AK, Rhodes A. What is a fluid challenge? Curr Opin Crit Care. 2011;17(3):290-5. 
30. Donati A, Carsetti A, Damiani E, Adrario E, Romano R, Pelaia P. Fluid responsiveness in critically ill patients. Indian J Crit Care Med. 2015;19(7):375-6.

31. Monnet X, Marik PE, Teboul JL. Prediction of fluid responsiveness: an update. Ann Intensive Care. 2016;6(1):111.

32. Vincent J-L. “Let's Give Some Fluid and See What Happens” versus the “Mini-fluid Challenge”. Anesthesiology. 2011;115:455-6.

33. Messina A, Dell'Anna A, Baggiani M, Torrini F, Maresca GM, Bennett V, Saderi L, Sotgiu G, Antonelli M, Cecconi M. Functional hemodynamic tests: a systematic review and a metanalysis on the reliability of the end-expiratory occlusion test and of the mini-fluid challenge in predicting fluid responsiveness. Crit Care. 2019;23(1):264.

34. Gotts JE, Matthay MA. Sepsis: pathophysiology and clinical management. BMJ. 2016;353:i1585.

35. Magder S. Volume and its relationship to cardiac output and venous return. Crit Care. 2016;20:271.

36. Gorrasi J, Pazos A, Florio L, Americo C, Lluberas N, Parma G, Lluberas R. Cardiac output measured by transthoracic echocardiography and Swan-Ganz catheter. A comparative study in mechanically ventilated patients with high positive end-expiratory pressure. Rev Bras Ter Intensiva. 2019;31(4):474-82.

37. Dohi TNS. Errors in the measurement of cardiac output by thermodilution. CAN JANAESTH. 1993;40:142-53.

38. Moller-Sorensen H, Graeser K, Hansen KL, Zemtsovski M, Sander EM, Nilsson JC. Measurements of cardiac output obtained with transesophageal echocardiography and pulmonary artery thermodilution are not interchangeable. Acta Anaesthesiol Scand. 2014;58(1):80-8.

39. Stetz CW, Kelly RGM,GE, Raffin TA. Reliability of the Thermodilution Method in the Determination of Cardiac Output in Clinical Practice. 1982.

40. Sasse SA, Berry PAC,RB, Sassoon CS, Mahutte CK. Variability of cardiac output over time in medical intensive care unit patients. Crit Care Med 1994.

41. Nadeau S. WHN: Limitations of cardiac output measurements by thermodilution. Can Anaesth Soc J 1986 Nov; 33(6):780-784.

42. Velissaris D, Pierrakos C, Scolletta S, De Backer D, Vincent JL. High mixed venous oxygen saturation levels do not exclude fluid responsiveness in critically ill septic patients. Crit Care. 2011;15(4):R177.

43. Rivers E, Nguyen B, Havstad S, Ressler J, Muzzin A, Knoblich B, Peterson E, Tomlanovich M. Early Goal-Directed Therapy Collaborative G: Early goal-directed therapy in the treatment of severe sepsis and septic shock. N Engl J Med. 2001;345(19):1368-77.

44. Lester AH, Critchley JAJHC. A meta-analysis of studies using bias and precision statistics to compare cardiac output measurement techniques. J Clin Monit Comput. 1999;15:85-91.

45. Toscani L, Aya HD, Antonakaki D, Bastoni D, Watson X, Arulkumaran N, Rhodes A, Cecconi M. What is the impact of the fluid challenge technique on diagnosis of fluid responsiveness? A systematic review and meta-analysis. Crit Care. 2017;21(1):207.

\section{Figures}




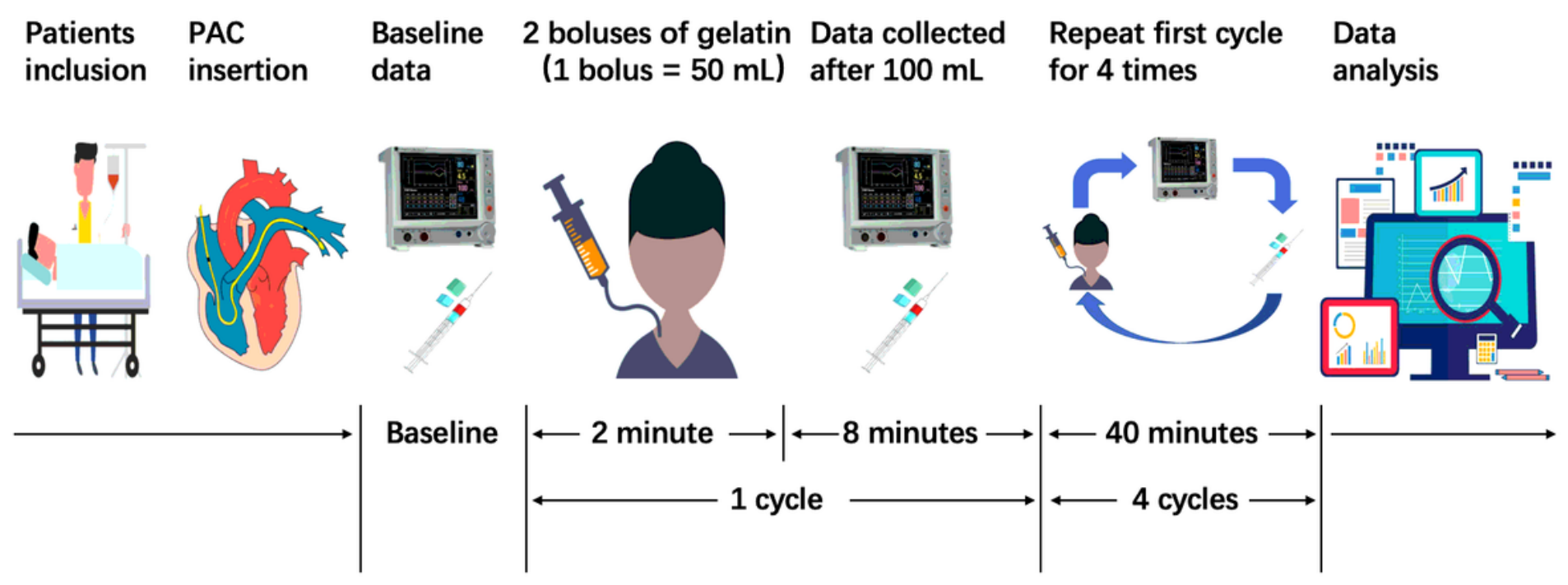

Fig.1 Study design.

Figure 1

Study Design 


\section{Study flow chart}

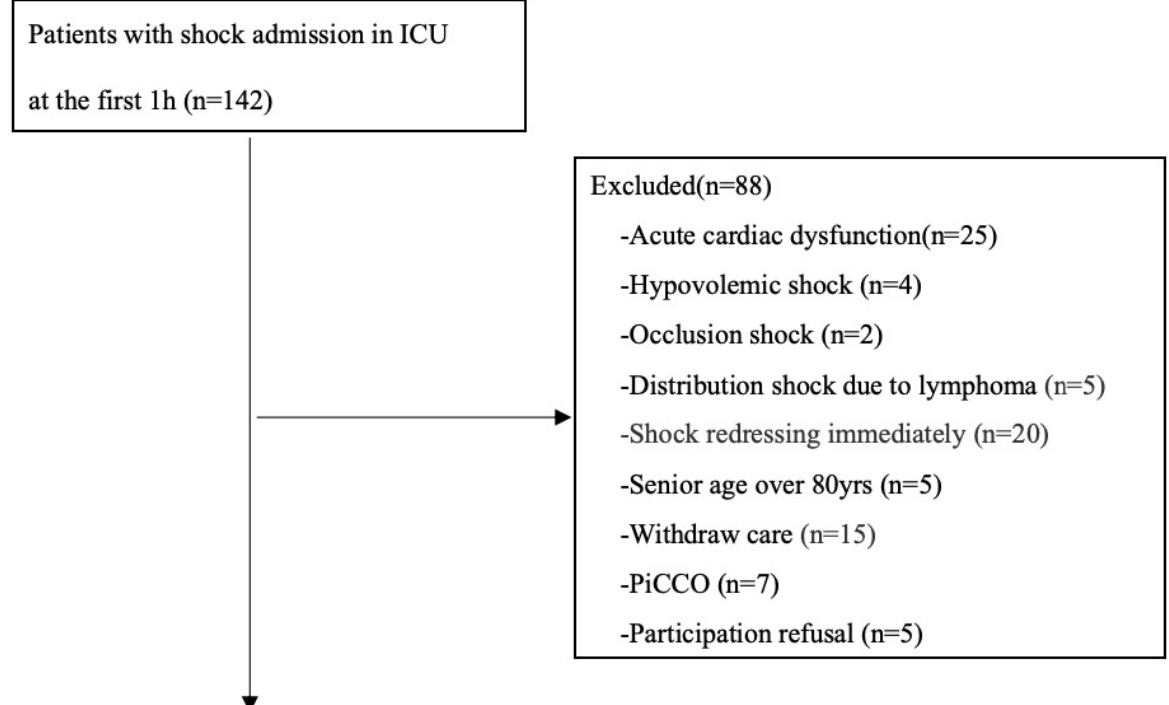

Before fluid challenge, completing PAC

monitoring $(\mathrm{n}=54)$

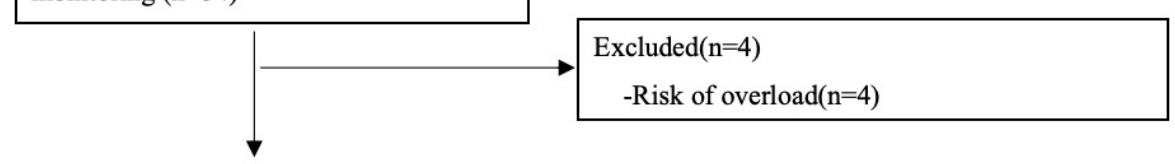

Mini-fluid challenge $(\mathrm{n}=50)$

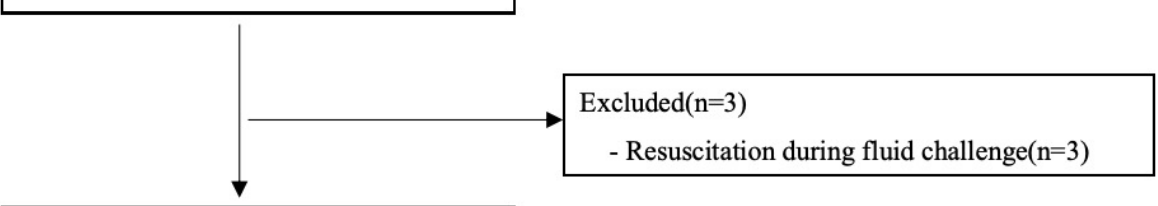

Included in final analysis $(\mathrm{n}=47)$

Fig. 2 Study flow chart

\section{Figure 2}

Study Flow

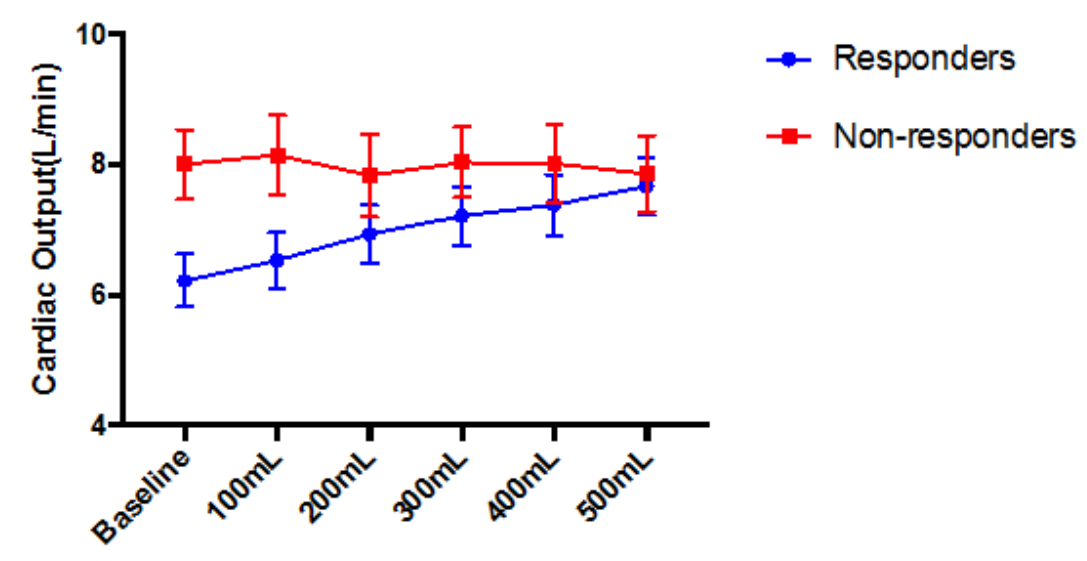

\section{Figure 3}

The tendency of $\mathrm{CO}$ after mini-fluid challenge in positive and negative responders. 


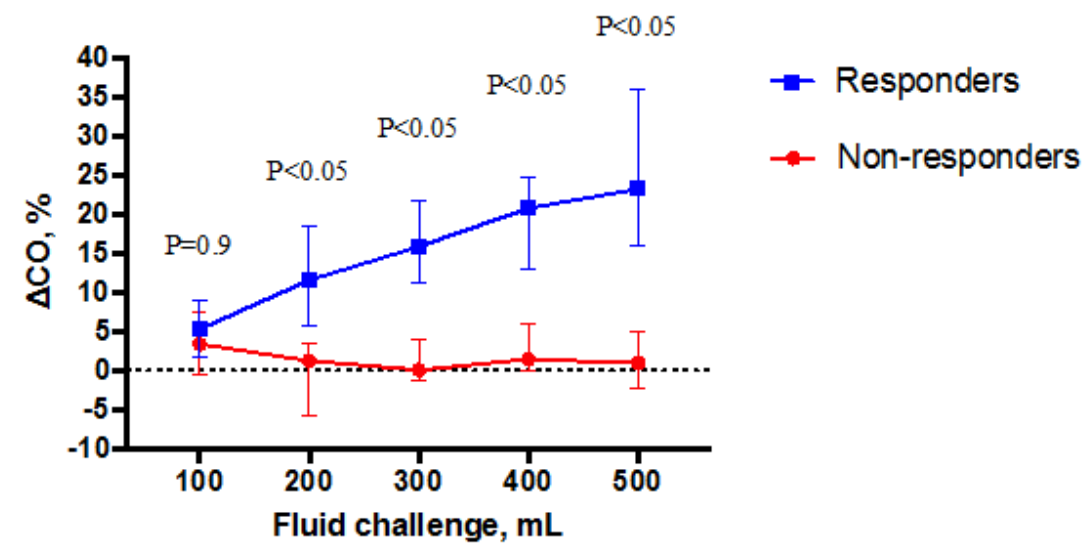

Figure 4

The median increase in cardiac output according to a fluid response defined by an increase in $\mathrm{CO} \geq 10 \%$ after $500 \mathrm{~mL}$ fluid infusion with $\mathrm{P}$ values (Mann-Whitney U test).
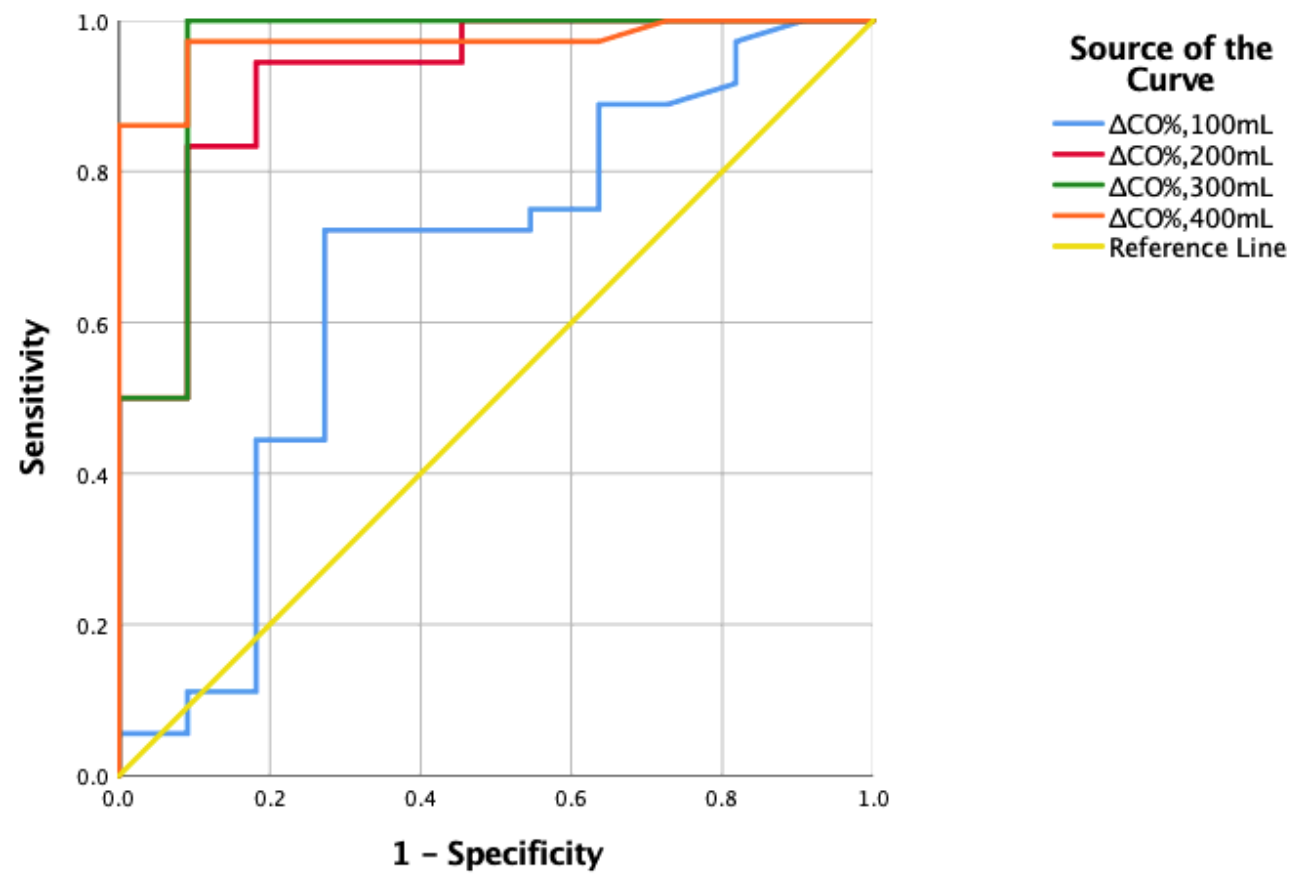

Figure 5

Receiver operating characteristics curves generated for mini-fluid challenge. $\triangle \mathrm{CO} \%, 100 \mathrm{~mL}$ (changes in cardiac output induced by a rapid $100 \mathrm{~mL}$ volume expansion). $\Delta \mathrm{CO} \%, 200 \mathrm{~mL}$ (changes in cardiac output induced by an accumulative of $200 \mathrm{~mL}$ volume expansion). $\Delta \mathrm{CO} \%$, $300 \mathrm{~mL}$ and $\Delta \mathrm{CO} \%, 400 \mathrm{~mL}$ in the same definition showing its abilities to predict positive fluid responsiveness. Positive fluid challenge was defined as an increase in cardiac output greater than $10 \%$ from baseline after an infusion of $500 \mathrm{~mL}$. 

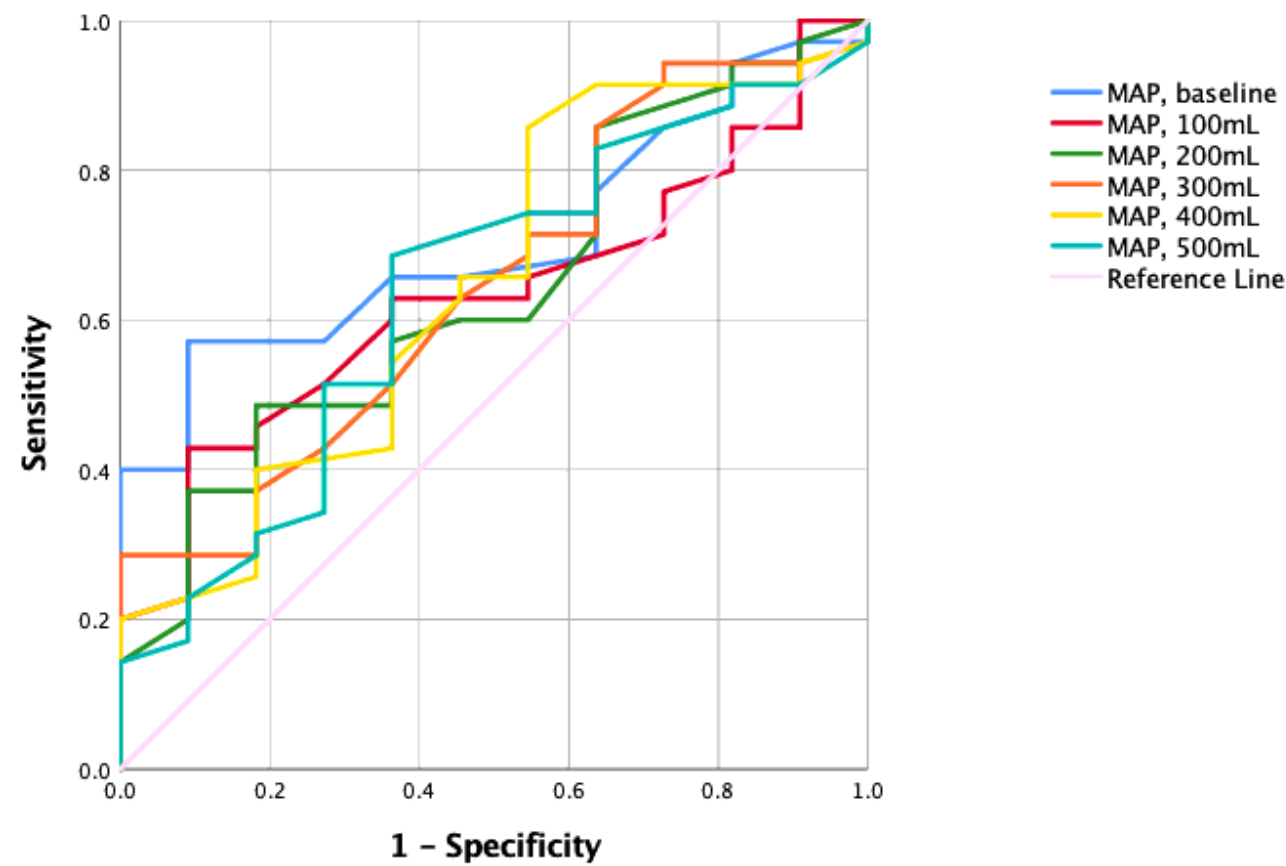

Figure 6

Receiver operating characteristic curve of MAPbaseline, MAP100, MAP200, MAP300, MAP400 MAP500 to predict fluid responsiveness. MAP at baseline has predictive power with AUC $0.71(\mathrm{p}<0.05,95 \% \mathrm{Cl}: 0.56-0.86)$, the best cut-off is $61 \mathrm{mmHg}$ with a sensitivity and specificity of $100 \%$ and $100 \%$.

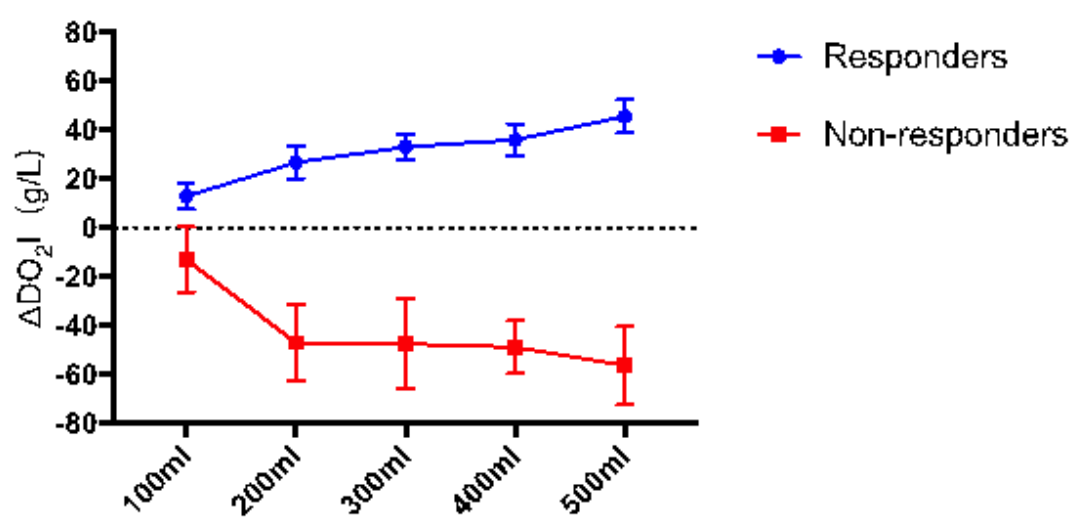

Figure 7

The tendency of $\triangle \mathrm{DO} 2 \mathrm{l}$ after mini-fluid challenge in R and NR. 


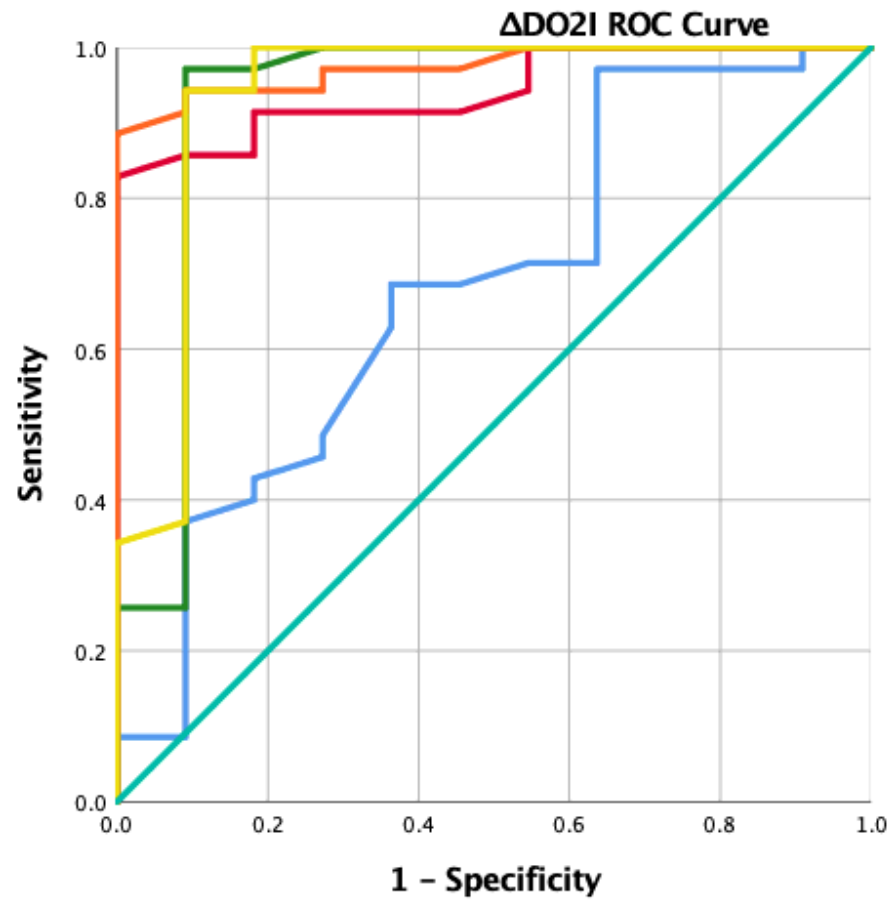

\section{Source of the \\ Curve \\ $\triangle \mathrm{DO} 2 \mathrm{l} 100 \mathrm{ml}$-base \\ $-\triangle \mathrm{DO} 2 \mathrm{I} 200 \mathrm{ml}$-base \\ $\triangle \mathrm{DO} 2 \mathrm{3} 300 \mathrm{ml}$-base \\ $\triangle \mathrm{DO} 2 \mathrm{l} 400 \mathrm{ml}$-base \\ $\triangle \mathrm{DO} 2 \mathrm{l} 500 \mathrm{ml}$-base \\ Reference Line}

Figure 8

Receiver operating characteristic curve of $\Delta D 021100, \Delta D 021200, \Delta D 021300, \Delta D 021400 \Delta D 02 I 500$ to predict fluid responsiveness

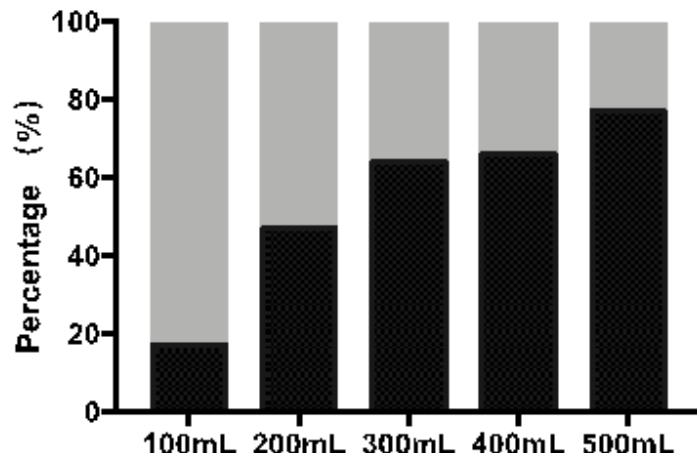

Nonresponders

Rsponders

Fluid challenge, $\mathrm{mL}$

Figure 9

FR were defined as $\mathrm{CO}>10 \%$ at each volume of FC, proportion of responders increased with the amount used for a fluid challenge.

Proportions were compared across different volume of colloid with a chi-square statistic. The $P$ values was significantly different ( $p<0.05)$. 